\title{
Reaction rates and effective parameters in stratified aquifers
}

\author{
Daniel Fernàndez-Garcia ${ }^{\mathrm{a}, *}$, Xavier Sánchez-Vila ${ }^{\mathrm{a}}$, Alberto Guadagnini ${ }^{\mathrm{b}}$ \\ a Department of Geotechnical Engineering and Geosciences, Technical University of Catalonia, C/Jordi Girona 1-3, 08034 Barcelona, Spain \\ ${ }^{\mathrm{b}}$ Dipartimento di Ingegneria Idraulica, Ambientale, Infrastrutture Viarie, Rilevamento, Politecnico di Milano, Milan, Italy
}

\section{A R T I C L E I N F O}

\section{Article history:}

Received 10 January 2008

Received in revised form 17 June 2008

Accepted 1 July 2008

Available online 5 July 2008

\section{Keywords:}

Multispecies reactive transport

Reaction rates

Stochastic hydrogeology

Heterogeneity

\begin{abstract}
A B S T R A C T
Chemical species are advected by water and undergo mixing processes due to effects of local diffusion and/or dispersion. In turn, mixing causes reactions to take place so that the system can locally equilibrate. In general, a multicomponent reactive transport problem is described through a system of coupled nonlinear partial differential equations. Under instantaneous chemical equilibrium, a complex geochemical problem can be highly simplified by fully defining the system in terms of conservative quantities, termed master species or components, and the space-time distribution of reaction rates. We investigate the parameters controlling reaction rates in a heterogeneous aquifer at short distances from the source. Hydraulic conductivity at this scale is modeled as a random process with highly anisotropic correlation structure. In the limit for very large horizontal integral scales, the medium can be considered as stratified. Upon modeling transport by means of an ADE (Advection Dispersion Equation), we derive closed-form analytical solutions for statistical moments of reaction rates for the particular case of negligible transverse dispersion. This allows obtaining an expression for an effective hydraulic conductivity, $K_{\mathrm{eff}}^{\mathrm{R}}$, as a representative parameter describing the mean behavior of the reactive system. The resulting $K_{\mathrm{eff}}^{\mathrm{R}}$ is significantly smaller than the effective conductivity representative of the flow problem. Finally, we analyze numerically the effect of accounting for transverse local dispersion. We show that transverse dispersion causes no variation in the distribution of (ensemble) moments of local reaction rates at very short travel times, while it becomes the dominant effect for intermediate to large travel times.
\end{abstract}

(c) 2008 Elsevier Ltd. All rights reserved.

\section{Introduction}

The potential risk associated with polluted groundwater systems depends on the rate at which chemicals displace and interact through an aquifer. Major challenges associated with modeling flow and reactive transport in naturally heterogeneous porous media include the incorporation of the effect of physical heterogeneity into multicomponent geochemical models and the quantification of its effects on the resulting spatially variable reaction rates. This is typically accomplished, for instance, by means of reactive transport codes that can handle several species with different types of reactions (e.g., [34] and references included therein). The application of these codes is typically associated with computational burden and numerical problems stemming from the required accurate description of heterogeneity in complex geochemical systems. De Simoni et al. $[9,10]$ have proposed a methodology which partially overcomes these numerical problems. Their main result is an exact expression for the space-time distribution of the reaction rate as a function of the spatial distribution of the conservative components. In this context, the reaction rates of a

\footnotetext{
* Corresponding author. Tel.: +34934011698.

E-mail addresses: daniel.fernandez.g@upc.edu (D. Fernàndez-Garcia), xavier.sanchez-vila@upc.edu (X. Sánchez-Vila), alberto.guadagnini@polimi.it (A. Guadagnini).
}

complex multispecies reactive transport problem, which can typically be accomplished numerically, can be obtained by solving two independent problems: (a) the transport of one or more conservative species (termed components), and (b) a chemical speciation. The solution to each one of these problems is simpler than the solution of the complete coupled problem but, still, there is a need for some computing power.

The application of all these models requires that the spatial distribution of heterogeneous aquifer properties (e.g., hydraulic conductivity) is known for the investigated domain. Due to the scarce knowledge of the features of a geological formation, it has become common to treat spatially varying subsurface flow parameters as auto-correlated random fields (e.g., [6,15,32,21]). This, together with uncertainty in forcing terms (initial and boundary conditions and sources), renders the groundwater flow and transport equations stochastic (e.g., [8]).

A number of existing stochastic analyses of reacting transport in heterogeneous formations are mainly concerned with (or limited to) the prediction of spatial moments of plumes composed by (eventually kinetically) sorbing solutes within spatially varying velocity fields (e.g., $[17,29,31]$ ) and are mostly aimed at deriving effective and/or upscaled quantities describing the spreading and shape of an evolving contaminant plume. Other works consider the combined effects of physical and chemical heterogeneity on 
solutes residence times in the context of sorption-desorption processes (e.g., $[2,30,5,22,37,14]$ ) or study the effects of micro-scale heterogeneous reactions on effective mass transfer parameters $[11,28,23,26]$. In all these studies, either analytical or Monte-Carlo based, a key point is that the groundwater velocity field can be conveniently represented in terms of a rescaled (or retarded) velocity.

Adopting the conceptual framework of De Simoni et al. $[9,10]$ can help to extend theoretical stochastic analysis of reactive transport in randomly heterogeneous aquifers to include different types of reactions, e.g., precipitation/dissolution. A recent application is provided by Luo et al. [24], who presented a macroscopic formulation of reaction parameters using small-perturbation theory. Here, our key objective is to provide and extension of the results of De Simoni et al. [9] to obtain exact analytical/numerical predictions and associated uncertainty of reaction rates in randomly stratified heterogeneous media. Our solution directly relates the geostatistical descrintors of the aquifer hydraulic conductivity and the chemistry-related parameters to the spatial distribution of the reaction rate. The latter is in turn characterized by its (ensemble) moments.

We consider one of the simplest conceptual models of heterogeneity, which is that of a stratified formation. According to this model, hydraulic conductivity, $K$, varies only in the vertical direction, $z$. Interest in this model has been motivated by its simplicity and by the recognition of the importance of layering on solute transport in sedimentary formations (e.g., $[27,25,4]$ ). Its simplicity

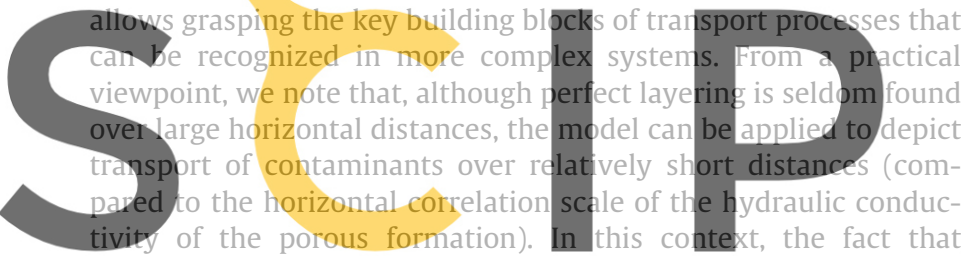
concentration gradients are usually higher close to a contaminant source implies that most mixing-driven chemical reactions take

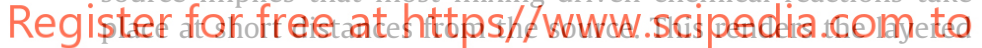
a suitable model to grasp key aspects associated with the examined processes. Moreover, the model also provides insight on the transport processes taking place in heterogeneous fields with high correlation of extreme $K$ values. The interest in this model stems from the observation that well connected geologic media with high degrees of heterogeneity have been observed to exhibit characteristics similar to that of a layered system [40]. Therefore, the concept of short distances is relative, and can be valid depending on the actual aquifer, from a few centimeters to a tens of meters or more.

Since our interest is in processes, we select a rather simple geochemical problem. We analyze a bi-molecular system where two aqueous solutes are in chemical equilibrium with an immobile solid mineral and undergo instantaneous precipitation. This is the case for instance of gypsum dissolution/precipitation observed in sedimentary aquifers due to mixing of waters with different chemical compositions. Update of permeability as a consequence of mineral clogging is disregarded. We emphasize that this system cannot be described in terms of a retardation coefficient which would eventually depend on a spatially heterogeneous distribution coefficient.

\section{Problem description}

\subsection{Problem statement and flow set-up configuration}

Reactions take place when a system is not in chemical equilibrium. This can be caused by different processes, including advection and dispersion. Advection becomes a relevant process only if reactions are not in instantaneous equilibrium. In such a case a water which is not in equilibrium with a mineral will move carrying a reaction force along its path. When the characteristic advective time is larger than the characteristic reaction time, reactions will not be driven by advective processes and instantaneous equilibrium can be assumed to hold at all points in the domain. In this

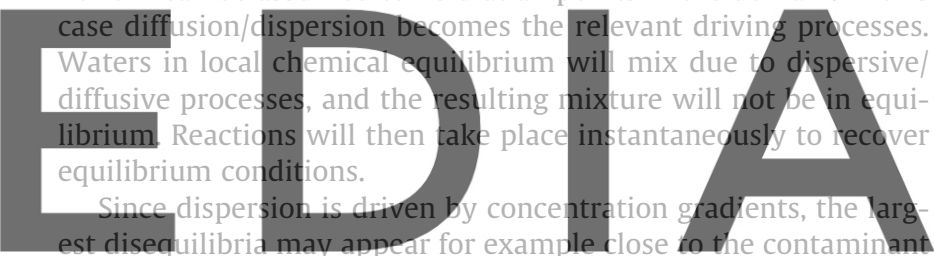

source in the case of a pulse injection. In this case, while dispersion will help increasing the affected reactive volume, the largest gradi

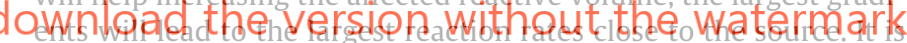

then appropriate to focus on the assessment of the reactions that would take place relatively close to the injection source.

We consider the problem set-up of Fig. 1, consisting of a sedimentary alluvial deposit conceptualized as a stratified system. Hydraulic conductivity is only a function of the (vertical) $z$-coordinate, $K(z)$, and under uniform gradient, $\mathbf{J}=(J, 0,0)$, with $J>0$, flow takes place only along the horizontal $x$-axis and is locally proportional to $K$, according to Darcy's law, $q_{x}(z)=K(z) J, q_{x}(z)$ being

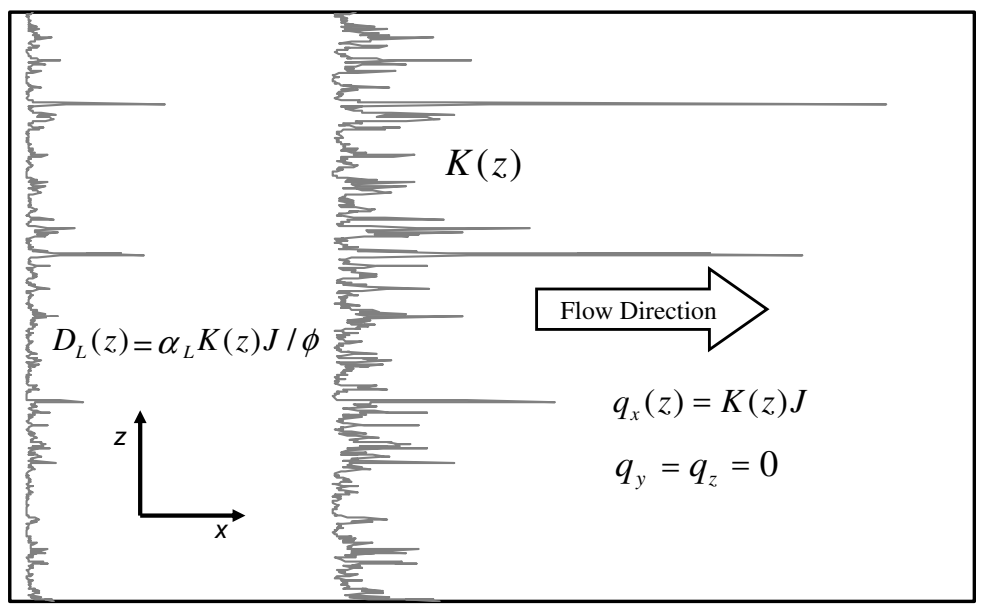

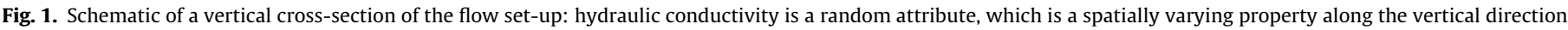
with perfect horizontal correlation, i.e., $K=K(z)$, and hydraulic gradient is oriented parallel to layering, $\mathbf{J}=(J, 0,0)$. 
Darcy's flux component along the $x$-direction. We consider the domain as infinite so that solute transport is not influenced by the presence of boundaries. Heterogeneity is only due to the spatial variability of hydraulic conductivity. The latter is modeled as a correlated random space function with a univariate log-normal distribution $\left(Y=\ln K \sim N\left(\mu, \sigma_{Y}^{2}\right), \mu\right.$ and $\sigma_{Y}^{2}$ respectively being the ensemble mean and variance of $Y$ ) and a very large horizontal integral scale $\lambda_{\mathrm{H}}$. Since we are interested in short travel distances $\left(x \ll \lambda_{\mathrm{H}}\right)$, we will consider for all operational purposes $\lambda_{\mathrm{H}} \rightarrow \infty$. The vertical integral scale, $\lambda_{z}$, is finite and significantly short when compared to the input source size.

The set-up described can be conceptually seen as that of an aquifer where vertical layers transition is smooth and no clear facies transitions are identifiable. Thus, it should not be seen as a stratified aquifer in the typical sense, i.e., in the presence of clearly identifiable layering.

\subsection{Multisnecies reactive transport problem}

We consider a dissolution/precipitation problem involving the mixing of two different waters. Each water carries in solution two aqueous species, $B_{1}$ and $B_{2}$, in instantaneous local equilibrium with a solid mineral $M_{3}$. The corresponding reaction is

\section{$v_{1} B_{1}+v_{2} B_{2} \rightleftarrows M_{3}$}

Without any loss of generality in the procedure for the solution of multicomponent reactive transport we will consider $v_{1}=v_{2}=1$.
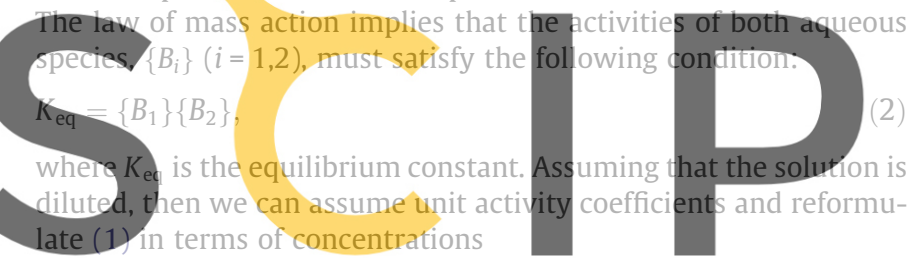

$K=C_{1} C_{2}$

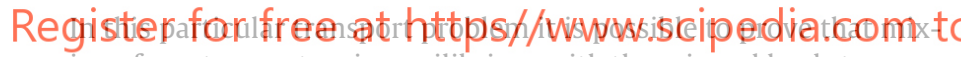
ing of any two waters in equilibrium with the mineral leads to oversaturation of the resulting mixture. Precipitation then takes place instantaneousiy in order to re-equilibrate the system. This wili not necessarily hold for more complex chemical systems, where mixing can lead to undersaturation and thus drive dissolution processes.

The geochemical problem can also be described by introducing the concepts of end-members and mixing ratios [3]. An end-member is a water characterized by a given geochemical signature, which is supposed to be in equilibrium with the mineral. Mixing ratios denote the fraction of a particular end-member that takes place in a mixture (in the case of non-reactive systems, the composition of any mixture is a linear combination of end-members). A geochemical system in equilibrium is fully characterized in terms of mixing ratios or, alternatively, in terms of conservative components (linear combinations of aqueous species concentrations not affected by reactions). Here, we used the methodology of De Simoni et al. [9] and thereby we determine reaction rates by means of conservative components.

\subsection{Transport model and computation of local reaction rates}

The mass balance equations for the two aqueous species are

$\phi \frac{\partial c_{i}}{\partial t}=-K(z) J \frac{\partial c_{i}}{\partial x}+\nabla \cdot\left(\phi \mathbf{D} \nabla c_{i}\right)-\phi r, \quad i=1,2$,

subject to the corresponding boundary and initial conditions. In (4), $\phi[-]$ is porosity which we take as constant, $\mathbf{D}\left[L^{2} T^{-1}\right]$ is the hydrodynamic dispersion tensor, and $r$ is a sink/source term $\left[M L^{-3} T^{-1}\right]$ that accounts for the solute removed from the system by precipitation (i.e., precipitated mass per unit fluid volume and time).

The system can be fully defined by means of a conservative concentration component, $u$, defined as

$u=c_{1}-c_{2}$.

The fact that $u$ is conservative stems from subtracting the two equations written in (4), leading to

$\phi \frac{\partial u}{\partial t}=-K(z) J \frac{\partial u}{\partial x}+\nabla \cdot(\phi \mathbf{D} \nabla u)$

where obviously the sink/source non-linear term does not appear. Eq. (6) can then be solved with the corresponding initial and boundary conditions in order to obtain $u$. Once $u$ is obtained, then the species concentrations can be explicitly computed as

$c_{1}=\frac{u+\sqrt{u^{2}+4 K_{\mathrm{eq}}}}{2}, \quad c_{2}=\frac{-u+\sqrt{u^{2}+4 K_{\mathrm{eq}}}}{2}$.

By applying the chain rule to Eq. (4), assuming that $K_{\text {eq }}$ is uniform in space and time, and incorporating (6), De Simoni et al. [9] found an explicit expression for the reaction rate

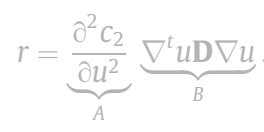

In (8), the $B$ term is a measure of dilution taking place by mixing, while the $A$ term is directly associated with the chemistry of the system and has an explicit expression in terms of $u$. In the particular $\left.\frac{\partial^{2} c_{2}}{\partial u^{2}}=\frac{2 K_{\mathrm{eq}}}{\left(u^{2}\right.}+4 K_{\mathrm{eq}}\right)^{3 / 2}$
In short, it follows from (8) and (9) that $r=f(u)$. This allows to
explicitly calculate $r$ at any given point in the domain and time only by solving a conservative transport problem. In general, for a different chemical system (arbitrary stoichiometric and activity coeffi-

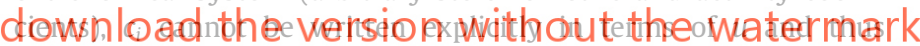
$\partial^{2} c_{2} / \partial u^{2}$ must be obtained indirectly through an implicit approach. Also, we note that (8) relies on the assumption that the advectiondispersion equation (ADE) adequateiy describes transport at some scale of interest (Darcy-scale). If a different model for transport is adopted, the general methodology is still valid but the resulting expression for the reaction rate will be different.

\section{Analytical solution for negligible transverse dispersion}

\subsection{Local reaction rates}

In the case of negligible transverse diffusion and dispersion, the reactive transport problem posed in Section 2.3 can be fully solved. We consider an aquifer which is initially under conditions of homogeneous geochemical equilibrium at all points, that is

$c_{i}(x, z, t=0)=c_{i, 0}, \quad i=1,2$,

where $c_{i, 0}$ satisfy (3). This is equivalent to stating that $u(x, z, t=0)=u_{0}=c_{1,0}-c_{2,0}$. We then inject instantaneously along a given line perpendicular to the flow direction a water with a different chemical composition (in our notation a different end-member). This end-member is also in chemical equilibrium with the mineral. Writing the initial conditions directly in terms of $u$

$u\left(x, z, t=0^{+}\right)=u_{0}+\Delta u_{0} \delta(x)$,

$\delta$ being the Dirac delta function. It should be clear that $\Delta u_{0}=\Delta c_{1,0}-\Delta c_{2,0}$ is not an actual concentration (it could even be negative). It represents a convenient way to state that the injected water has a different chemical signature than the resident one. We 
start by assuming that mass is uniformly introduced in the system along the injection line, independently of local velocity values. Then, we discuss the solution for the scenario corresponding to an injection proportional to local fluxes in Section 3.5.

The presence of the two end-members produces a chemical disequilibrium through mixing. The input water is advected, and in the absence of transverse diffusion and dispersion, the only mixing mechanism is that of longitudinal diffusion/dispersion, taking part at the moving boundary (initially sharp) between the waters. By its particular definition, $u$ is not affected by precipitation. Thus, we can write directly the solution of the transport problem as [1]

$u(x, K(z), t)=u_{0}+\frac{\Delta u_{0}}{\sqrt{4 \pi D_{\mathrm{L}}(K) t}} \exp \left(-\frac{(x-v(K) t)^{2}}{4 D_{\mathrm{L}}(K) t}\right)$.

In (12) we are explicitly stating that $K$ is the only term which governs vertical, i.e., along $z$, variability of $u$; longitudinal dispersion is written as $D_{\mathrm{L}}(K)=\alpha_{\mathrm{L}} K(z) J / \phi$ (without any loss of generality molecular diffusion was neglected) and groundwater velocity is $v(K)=K(z) J / \phi$. From (12), the dilution term in Eq. (8) is

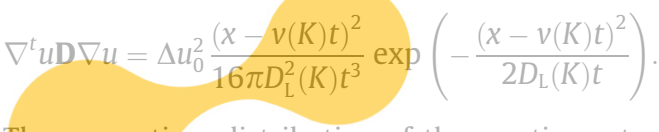

The space-time distribution of the reaction rate, $r(x, K, t)$, is then obtained by incorporating (9) and (13) into (8).

Since $K$ is a spatial random function, so is $r$. By assuming a particular distribution for $K$, we can obtain the statistical moments of $r$

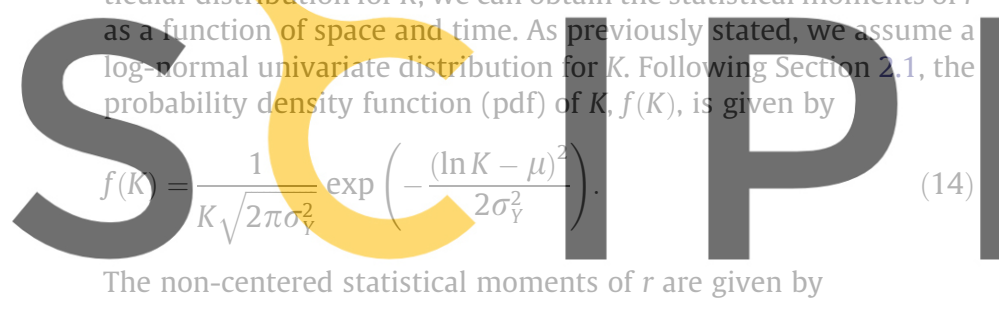

Registert) forf friee rat) frottprs//www.scipedia.com(to)

Here and in the following the overbar denotes the ensemble operator. The standard deviation of local reaction rates is defined as the square root of its variance, $\sigma_{r}^{2}=\overline{r^{2}}-\bar{r}^{2}$. Computation of these statistical moments involves only one quadrature and can be easily evaluated numerically.

Fig. 2a displays the local mean reaction rate for different values of the time elapsed since injection as a function of the advective travel time $t_{\mathrm{adv}}=x / V_{\mathrm{A}}\left(V_{\mathrm{A}}\right.$ being the advective velocity of a homogeneous medium characterized by an effective hydraulic conductivity equal to the arithmetic mean, $K_{\mathrm{A}}=\exp \left(\mu+\sigma_{Y}^{2} / 2\right)$, of the local conductivities of the randomly heterogeneous stratified system). Here, $\mu=0$ and $\sigma_{Y}^{2}=1$, while all other parameters adopted for the system are described in Table 1 . Results for the heterogeneous system are juxtaposed to those obtained for a homogeneous medium with $K=K_{\mathrm{A}}$.

The differences between the behavior of the solution for heterogeneous and homogeneous media are both qualitative and quantitative. As a term of comparison, Fig. 2b depicts the spatial distribution of reaction rates in an"equivalent" homogeneous medium characterized by a uniform velocity field, $V_{\mathrm{A}}=K_{\mathrm{A}} \mathrm{J} / \phi$. The conservative quantity $u$ is carried by groundwater flow and travels with local velocity $V_{\mathrm{A}}$. The simultaneous action of dispersion causes a symmetric redistribution of $u$ about $V_{\mathrm{A}} t$ described by a Gaussian curve with standard deviation $\sigma=\sqrt{2 D_{\mathrm{L}} t}$. Thus, at $x=V_{\mathrm{A}} t$, the profile of $u$ displays a maximum where $\partial u / \partial x=0$, causing the local reaction rate to vanish. Because of the Gaussian shape, the maximum value of the dilution term $D_{\mathrm{L}}(\partial u / \partial x)^{2}$ takes place at $x_{m}=V_{\mathrm{A}} t \pm \sigma$. However, the impact of the speciation term
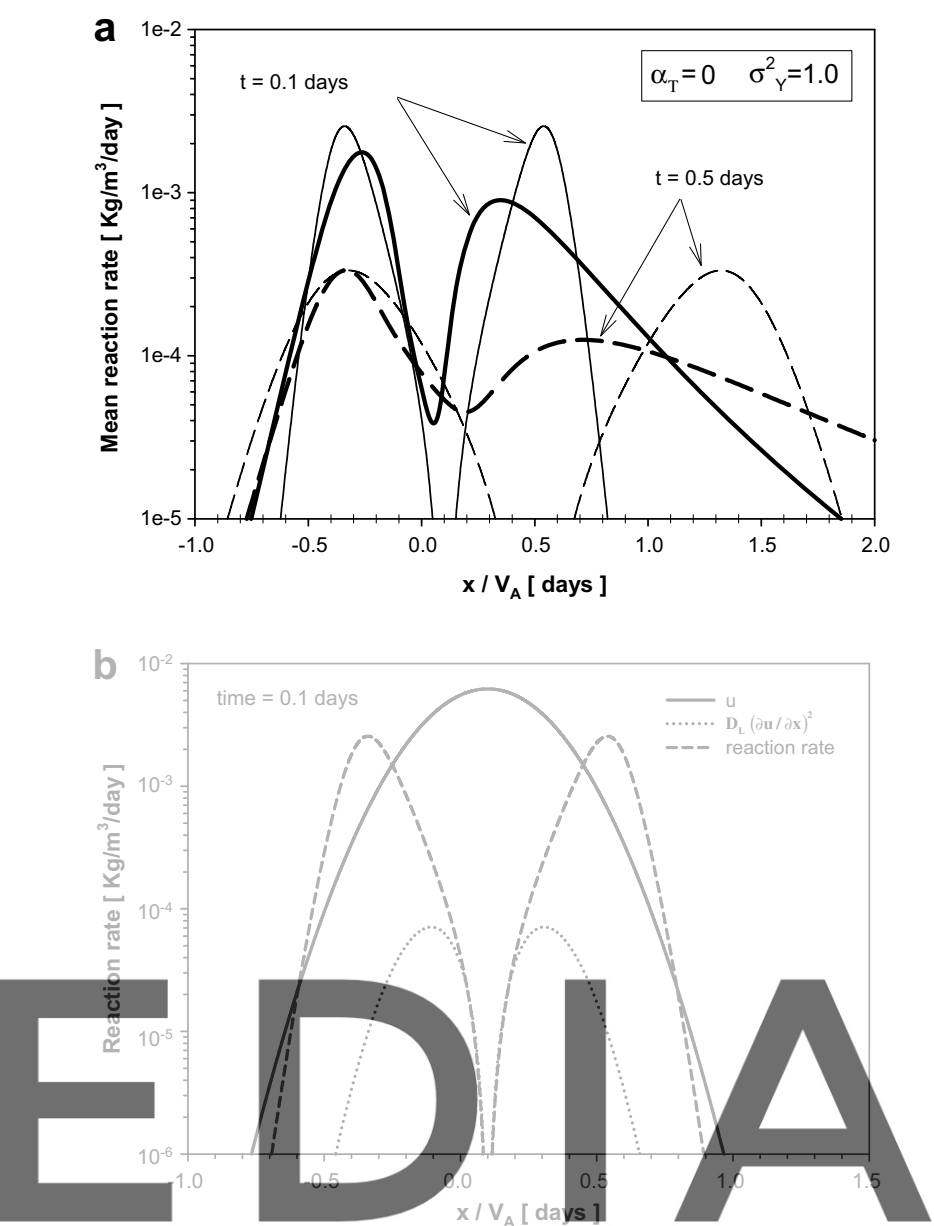

Fig. 2. (a) Mean reaction rate as a function of distance from the source for different

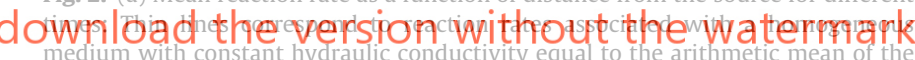
local hydraulic conductivities, $K_{A}$. Additional parameters are shown in Table 1; (b) Conservative component $u$, term $D_{\mathrm{L}}(\partial u / \partial x)^{2}$, and local reaction rate $r$ as a function of distance from the source in a homogeneous system after 0.1 days from the injection. The $x$-distance from the injection is normalized by the arithmetic mean of the velocity field, $V_{\mathrm{A}}$.

(9) displaces $x_{m}$ away from this location of the peak without affecting the symmetry. In the heterogeneous medium (Fig. 2a), the ensemble mean reaction rate exhibits four significant features: (i) its spatial distribution is non-symmetrical, displaying two local maxima; (ii) the peak values are located closer to the source than what can be observed in the homogeneous system; (iii) the local minimum observed between the two peaks does not vanish, as opposed to the homogeneous system, and is located closer to the injection source than that observed in the homogeneous medium; and (iv) a long tail is observed down-gradient.

The coefficient of variation, defined as the ratio of the standard deviation to the mean, is a measure of the relative uncertainty associated with predictions. The corresponding coefficient of variation (CV) associated with reaction rates is depicted in Fig. 3. Here it is relevant to observe that (a) uncertainty is not a monotonic function of distance from the injection, being generally largest at the tail and the front of the reaction rate profile; and (b) the overall uncertainty associated with reaction rates decreases with time. In any case, most CV values are large, indicating the inability of the mean reaction rate to capture the key features of precipitation in the system, and the need to account for uncertainty in all cases. 
Table 1

Numerical features and simulation parameters

\begin{tabular}{|c|c|c|}
\hline Parameter & Description & Value \\
\hline \multicolumn{3}{|c|}{ Discretization } \\
\hline$n_{z}$ & Number of layers & 800 \\
\hline$L_{x}$ & Domain size in $x$-direction & $15.0 \mathrm{~m}$ \\
\hline$L_{z}$ & Domain size in $z$-direction & $32.0 \mathrm{~m}$ \\
\hline$\Delta z$ & Layer thickness & $0.04 \mathrm{~m}$ \\
\hline \multicolumn{3}{|c|}{ In $K$ random function } \\
\hline Model & Exponential & \\
\hline$K_{\mathrm{G}}$ & Geometric mean & $1.0 \mathrm{~m} /$ day \\
\hline$\lambda_{\mathrm{H}}$ & Integral scale in the horizontal plane & $\infty$ \\
\hline$\lambda_{z}$ & Integral scale in the $z$-direction & $0.2 \mathrm{~m}$ \\
\hline$\lambda_{z} / \Delta z$ & Resolution & 5 \\
\hline \multicolumn{3}{|c|}{ Transport parameters } \\
\hline$\phi$ & Porosity & 0.3 \\
\hline$J$ & Hydraulic gradient & 0.05 \\
\hline$\alpha_{\mathrm{L}}$ & Longitudinal dispersivity & $0.06 \mathrm{~m}$ \\
\hline$u_{0}$ & Initial concentration of $u=c_{1,0}-c_{2,0}$ & $0.0 \mathrm{~kg} / \mathrm{m}^{3}$ \\
\hline$\Delta u_{0}$ & Pulse injection of $u=c_{1,0}-c_{2,0}$ & $9 \times 10^{-4} \mathrm{~kg} / \mathrm{m}^{3}$ \\
\hline \multicolumn{3}{|c|}{ Chemical speciation } \\
\hline$K_{\mathrm{eq}}$ & Equilibrium constant $^{a}$ & $10^{-7}$ \\
\hline \multicolumn{3}{|c|}{ Particle tracking } \\
\hline$N_{p}$ & Number of particles & $10^{6}$ \\
\hline$\Delta t$ & Time step & $4 \times 10^{-3}$ days \\
\hline
\end{tabular}

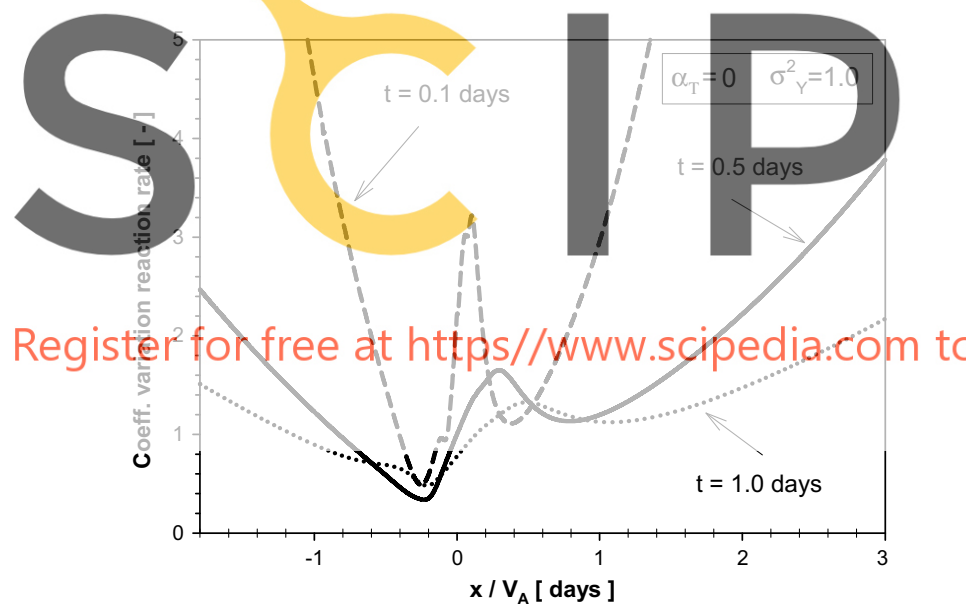

Fig. 3. Coefficient of variation of the reaction rate, $\mathrm{CV}=\sigma_{r}(x, t) / \bar{r}(x, t)$, as a function of distance from the source for different times. Same parameters used as in Fig. 2.

\subsection{Integrated and total reaction rates}

We first start by evaluating the (random) integrated reaction rate taking place in each particular layer, $R(K, t)$, defined as

$R(K, t)=\int_{-\infty}^{\infty} r(x, K, t) \mathrm{d} x$

We note that, according to our conceptual model for the heterogeneity of the system, a layer is actually represented by its corresponding $K$ value. In general, this integral must be evaluated numerically. Yet, an explicit approximation of (16) can be obtained whenever $u^{2}+4 K_{\mathrm{eq}}=u_{0}^{2}+4 K_{\mathrm{eq}}=$ constant, meaning that the chemical compositions of the injected and initial water are not very different. An evaluation of the actual range of validity is included in Appendix A. In this case the following expression holds:

$R(K, t)=\frac{\Delta u_{0}^{2} K_{\mathrm{eq}}}{4 \sqrt{2 \pi D_{\mathrm{L}}(K) t^{3}}\left(u_{0}^{2}+4 K_{\mathrm{eq}}\right)^{3 / 2}}=\frac{E}{\sqrt{K(z)}} \frac{1}{t^{3 / 2}}$, where $E$ is a constant coefficient that incorporates the contribution of $u_{0}, \Delta u_{0}, \alpha_{\mathrm{L}}, J, \phi$, and $K_{\text {eq }}$, and is given by

$E=\Delta u_{0}^{2} \frac{K_{\mathrm{eq}}}{4\left(u_{0}^{2}+4 K_{\mathrm{eq}}\right)^{3 / 2}}\left(2 \pi \alpha_{\mathrm{L}} \frac{J}{\phi}\right)^{-1 / 2}$.

We observe that the integrated reaction rate is a random function through $K$ and tend to decrease as $t^{-3 / 2}$. Furthermore, it can be noted that the fact that $R(K, t)$ is inversely proportional to $\sqrt{K(z)}$, implies that the smallest integrated reaction rates take place in the most conductive layers. This is associated with the fact that while on one side the effects of dispersion processes are large in the most conductive layers, on the other side large dispersions tend to reduce the gradients of $u$. The latter effect counteracts the former one and becomes dominant (see Eq. (8)). Interestingly, from (17), we note that the randomness of $R(K, t)$ directly stems from the randomness of the local dispersion coefficient $D_{\mathrm{L}}(K)$. This indicates that the usual assumption of neglecting local dispersion fluctuations in the study of conservative solute transport phenomena might not be an adequate approximation for evaluating reactive transport processes in heterogeneous media. In particular, for randomly stratified media with negligible transverse dispersion, if $\partial D_{\mathrm{L}}(K) / \partial K=0$ then the integrated reaction rate is deterministic while on the contrary the actual reaction rate value at any given point is still random.

The total mean reaction rate in the system $\bar{R}(t)$ is given then as $\bar{R}(t)=\int_{0}^{\infty} R(K, t) f(K) \mathrm{d} K$.

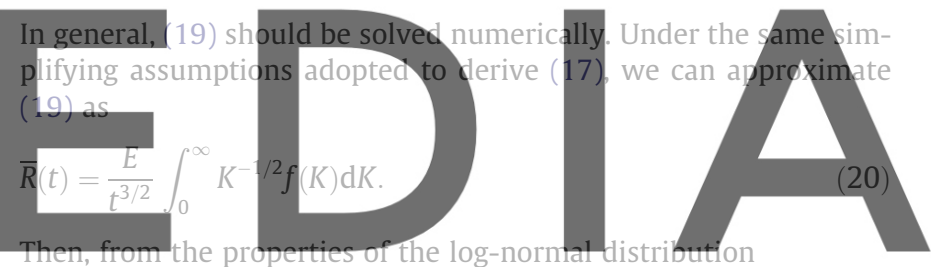

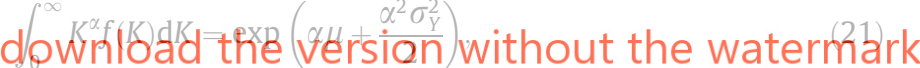
we obtain

$\bar{R}(t)=\frac{E}{t^{3 / 2}} \exp \left(-\frac{\mu}{2}+\frac{\sigma_{Y}^{2}}{8}\right)$

Fig. 4 compares the approximate expression (22) with the exact solution obtained by numerical integration of (16). As evaluated in Appendix A, the approximated solution is valid for large times

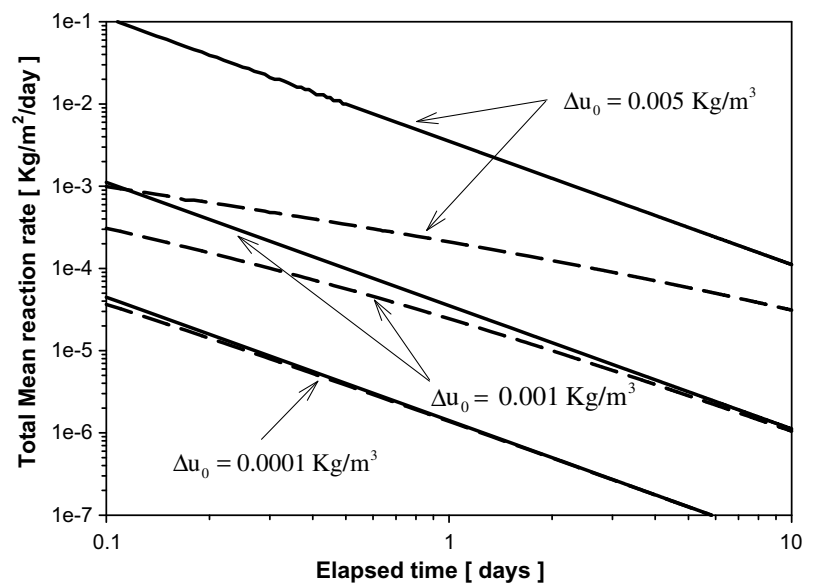

Fig. 4. Total mean reaction rate $\bar{R}(t)$ as a function of time elapsed since injection for different $\Delta u_{0}=c_{10}-c_{20}$ initial concentration values of the conservative component. Dashed lines are the values derived by numerically integrating (19). Solid lines represent the approximation (22). 
and small departures, $\Delta u_{0}$, of the injected water from the initial chemical composition (see Eqs. (43)-(47)). We note that the fact that the total reaction rate decreases with time as $t^{-3 / 2}$ is consistent with what observed by Luo et al. [24], albeit their approximate solution has been derived for a different type of heterogeneous medium, i.e., unconditional, randomly heterogeneous two-dimensional domain of infinite extent. Contrary to [24], in randomly stratified systems, there is no need for approximations and so, we provide an exact solution that includes the complete impact of heterogeneity, geochemical parameters, and local dispersion.

\subsection{Effective hydraulic conductivity value(s)}

Here we investigate the possibility of substituting the heterogeneous medium by a homogeneous system providing the same total mean reaction rate. Within this context, an effective conductivity $K_{\text {eff }}^{\mathrm{R}}$ can be defined as a direct extension of (17)

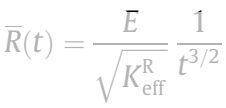

By direct comparison of (22) and (23), and from the properties of the log-normal distribution, it follows that:

$K_{\mathrm{eff}}^{\mathrm{R}}=\exp \left(\mu-\frac{\sigma_{Y}^{2}}{4}\right)=\sqrt{K_{\mathrm{H}} K_{\mathrm{G}}}$

so that the effective value defined by (23) lies halfway between the

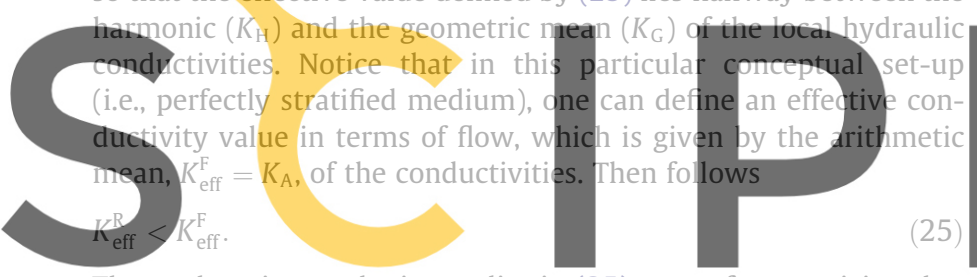

The explanation to the inequality in (25) stems from noticing that flow is controlled by the high conductive layers, while precipitation Registles fior freey atthatupsphinumivi.seipedia.com to We note that albeit this inequality is derived upon considering that all the randomness in $R(K, t)$ comes from $D_{\mathrm{L}}(K)$ (see Section 3.2), numericai simulations presented in Section 4 demonstrate that this discrepancy is even more important when the effects of transverse dispersion are taken into account.

\subsection{Standard deviation of integrated reaction rates}

The variance of the integrated reaction rate $R(K, t)$ can be computed as

$\sigma_{R}^{2}(t)=\overline{R^{2}}-\bar{R}^{2}$.

While the exact value can be computed numerically from (16), it is also possible to compute an approximation of (26) by means of (17). In the latter case, the resulting coefficient of variation can be written explicitly

$\frac{\sigma_{R}}{\bar{R}}=\left(\exp \left(\frac{\sigma_{Y}^{2}}{4}\right)-1\right)^{1 / 2}$

We note that this is quite a large value even for mild variances of $Y$. This suggests that predictive power associated with the mean integrated reaction rate for any given layer can be quite small. Higher order moments of $R$ could be obtained by using the same methodology.

\subsection{The impact of the choice of boundary conditions}

In order to analyze robustness of our solution, we study its sensitivity to the choice of boundary condition adopted for the transport problem. Therefore, we modify the boundary condition by allowing mass to enter the system proportionally to the water flux in each layer. This boundary condition is most suitable to model the entrance of solute mass into porous media by means of injection wells, when solute mass preferentially flows through the most conductive layers. Since the flux is proportional to $K$ in each layer, the solution of this particular problem is given by

$$
u(x, K(z), t)=u_{0}+\frac{K(z)}{K_{\mathrm{A}}} \frac{\Delta u_{0}}{\sqrt{4 \pi D_{\mathrm{L}}(K) t}} \exp \left(-\frac{(x-v(K) t)^{2}}{4 D_{\mathrm{L}}(K) t}\right) .
$$

Fig. 5 depicts the dependence of the mean reaction rate, $\bar{r}$, on the time elapsed since injection and the characteristic advective time $t_{\mathrm{A}}=x / V_{\mathrm{A}}$. Results associated with the injection mode adopted in Section 3.1, i.e., uniform distribution of solute mass along the injection line, are juxtaposed for comparing purposes. Since injection proportional to water flux allows most of the mass to be introduced in the high conducive regions, the initial concentration of $u$ is now distributed according to $u\left(x, z, t=0^{+}\right)=u_{0}+\Delta u_{0} \delta(x) K(z) / K_{\mathrm{A}}$, and reactions tend to be displaced further away from the injection. This also causes larger tailing in the curve. Furthermore, we note that, at small times, the peak and local minimum values observed in Fig. 5 are smaller when the injection is performed proportional to flux rather than in the case of uniform injection. This is a direct consequence of the fact that the largest reaction rates tend to take place in the less conducive areas and most of the solute mass is now in-

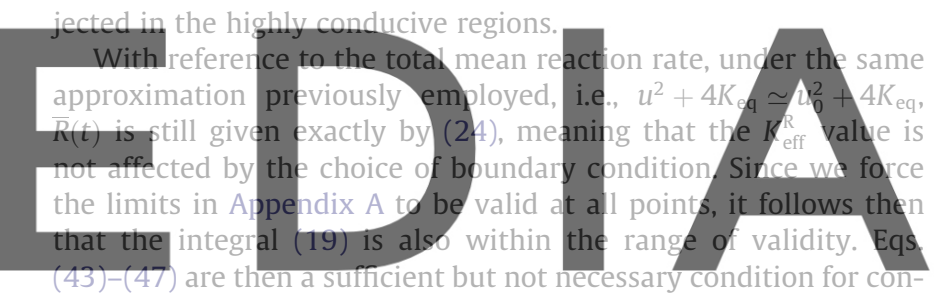

vergence. The behavior of this analytical result outside its range of

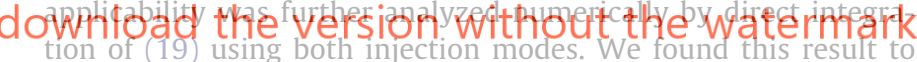
be valid beyond the limits of the approximation presented in Appendix A.

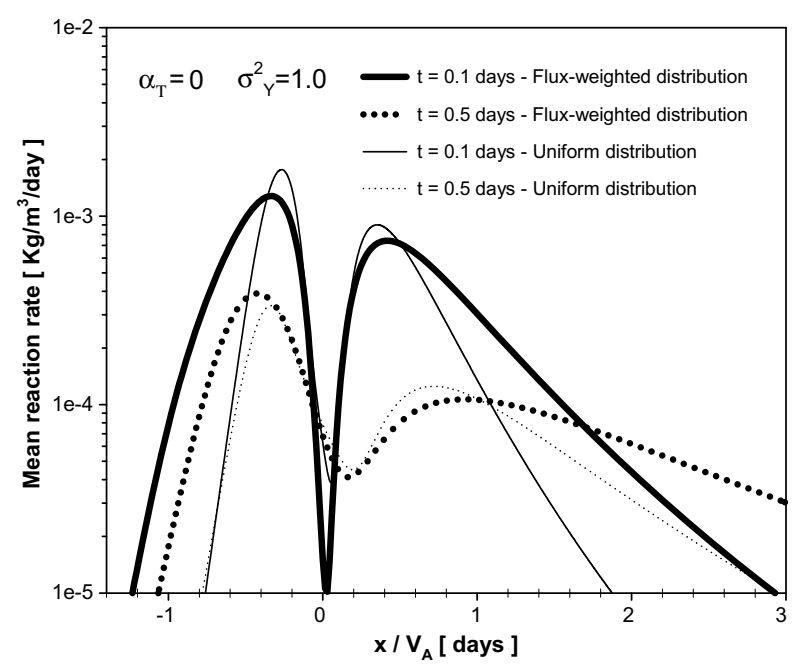

Fig. 5. Dependence of the ensemble mean reaction rate $\bar{r}(x, t)$, equation calculated according to (15), on distance and as a function of injection mode. The $x$-distance from injection is normalized by the arithmetic mean of the velocity field, $V_{\mathrm{A}}$. 


\section{Numerical simulations}

\subsection{Design of transport simulations}

Numerical simulations are aimed at two main objectives. On one hand they serve to illustrate that adopting the conceptual framework of De Simoni et al. [9] allows relatively simple solutions of a fairly complex multispecies reactive system; on the other hand, we employ numerical simulations to explore the effect of transverse dispersivity on reaction rates in a randomly heterogeneous stratified aquifer where closed-form solutions cannot be obtained. Simulations involve three distinct steps: First, we model solute transport of the conservative component $u(x, z, t)=$ $c_{1}(x, z, t)-c_{2}(x, z, t)$ in a prescribed stratified system using the Random Walk Particle Tracking code RW3D [13]. Then, we calculate local reaction rates of concentrations using the formulation of De Simoni et al. [9]. In this case, the explicit expression of $r$ is

$r(x, z, t)=r_{\mathrm{L}}+r_{\mathrm{T}}$

$$
\frac{\partial^{2} c_{2}}{\partial u^{2}}\left(\frac{\partial u}{\partial x}\right)^{2} \alpha_{L} \frac{K(z) J}{\phi}+\frac{\partial^{2} c_{2}}{\partial u^{2}}\left(\frac{\partial u}{\partial z}\right)^{2} \alpha_{T} \frac{K(z) J}{\phi}
$$

where $r_{\mathrm{L}}$ and $r_{\mathrm{T}}$ denote the longitudinal and transverse contribution to the reaction rate. Finally, we compute spatial moments of reaction rates, $\widehat{r}^{n}(x, t)(n=1,2)$ and $\widehat{R}(t)$, as

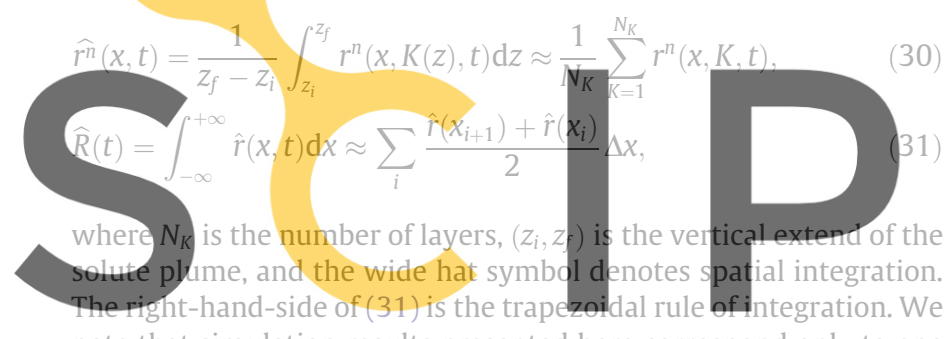
note that simulation results presented here correspond only to one Reindividual realization of the hydraylic conductivity random field

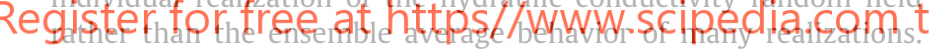

Nonetheless, as the given realization is provided with many vertical integral scales and the injection source size is much larger than the integral scale $\left(100 \lambda_{z}\right)$, it is reasonable to expect that low order spatial and statistical moments coincide, i.e., $\hat{r}(x, t) \approx \bar{r}(x, t)$ and $\widehat{R}(t) \approx \bar{R}(t)$. This will be further explored in Section 4.2.

A sketch of the set-up adopted for the conservative transport simulations is provided in Fig. 6. We consider a two-dimensional porous medium representing a vertical cross-section of a given stratified aquifer. Flow is driven by a uniform gradient oriented parallel to stratification ( $x$-axis in Fig. 6 ). The porous medium is formed by 800 layers, each layer having a different hydraulic conductivity value drawn from a log-normal distribution. All other layer properties remained spatially uniform. The selected log hydraulic conductivity distribution describes one arbitrary realization of a one-dimensional Gaussian random function following an exponential covariance function model, $C_{Y}\left(\left|h_{z}\right|\right)=\sigma_{Y}^{2} \exp \left(-\left|h_{z}\right| / \lambda_{z}\right)$, $h_{z}$ being the vertical lag separation distance. The variance of the $\ln K$-field and the geometric mean of the $K$-field are set to 1 $\left(K_{\mathrm{G}}=1, \sigma_{Y}^{2}=1.0\right)$. Numerical features and simulation parameters are summarized in Table 1.

The Random Walk Methodology was used to simulate the migration of a passive solute representing the component $u$. This methodology relies on splitting the plume into discrete mass particles and has the advantages of requiring small CPU times and being free of numerical dispersion. For each time step, particles were first allowed to move along flow lines by advection to subsequently experienced a Brownian motion (dispersion). For the system analyzed, this can be formally expressed as [35]

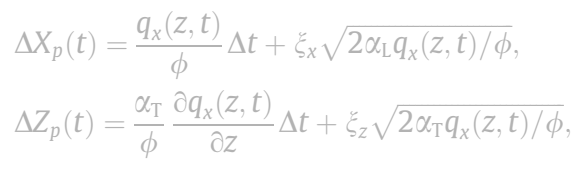

where $\Delta X_{p}(t)$ and $\Delta Z_{p}(t)$ are the particle displacements, $\Delta t$ is the time step, and $\xi_{x}$ and $\xi_{z}$ are two independent random variables described by standard normal distribution functions. Note that a cor-
rection term in the advective drift was added to addquate the
Fokker-Planck equation to its corresponding ADE [19]. The Random
Walk code used has been successfully applied in many differen
studies (e.g., [13,36]) and is provided with a hybrid scheme for
the interpolation of velocity that yields accurate mass/balances at
laver interfaces [35]. The transverse gradient of a was computed using finite difference. Initially, solute mass particles (1 Million) are unifprmly distributed along a line perpendjcular to the flow

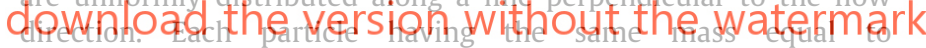
$m_{p}=\phi \Delta u_{0} \Delta z / N_{p}^{c}$, being $N_{p}^{c}$ the initial number of particles in one cell (2000) and $\Delta z$ the layer thickness. The line is located at mid-depth (Fig. 6) and extends 100 integral scales in the $z$ direction. The gap between this line and the limits of the domain measures over 25 vertical integral scales to prevent boundary effects.

We start by performing transport simulations of the conservative component in the absence of transverse dispersivity. This allows: (a) calibrating the number of particles needed in the

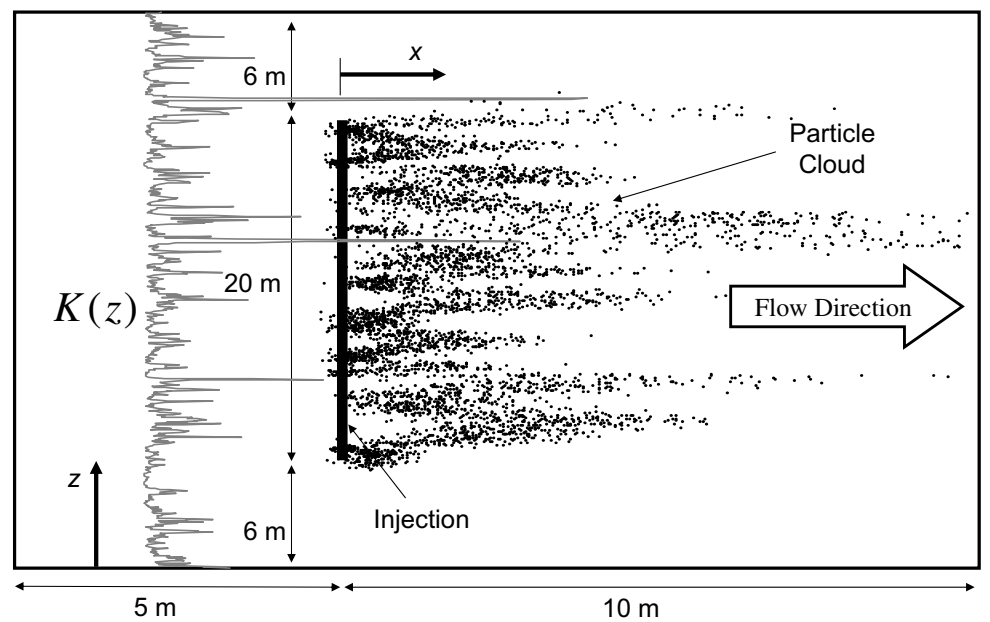

Fig. 6. Design of transport numerical simulations. 
simulations against the results of an (exact) analytical solution, and at the same time, (b) assessing the limitations of the ergodicy assumptions in our computations. These points will be further explored in Section 4.3. Then, the effect of transverse dispersion was investigated by executing the same simulation but using a transverse dispersivity equal to the longitudinal dispersivity. This choice of transverse dispersivity allows examining the impact of transverse dispersion on the reaction rates in the worse case scenario. Nonetheless, results are further applicable to other natural conditions as the effect of transverse mixing is measured in terms of the characteristic time of vertical mixing of a solute plume (see Section 4.3).

\subsection{Computation of local reaction rates and their moments}

As previously described, computation of local reaction rates $r(x, z, t)$ depends on the chemical speciation term $\partial^{2} c_{2} / \partial u^{2}$ (which is provided explicitly by (9) in this case), hydrodynamic dispersion, and the partial derivatives of the conservative component $\partial u / \partial x$ and $\partial u / \partial z$ (see Eq. (29)). We calculated these derivatives through forward finite differences, which requires accurate estimates of $u(x, z, t)$. Particle tracking techniques produce discrete distributions of the particle position at given times that have to be converted to a continuous distribution of concentrations. Smoothing techniques are typically employed for this matter. Essentially, concentrations are estimated as [39]

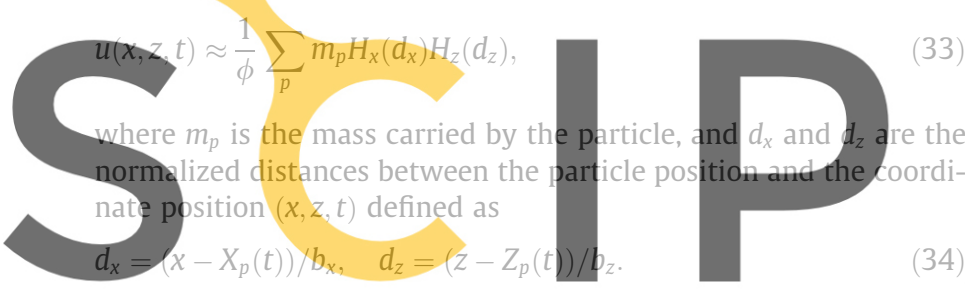

Here, $b_{x}$ and $b_{z}$ define the size of the support volume over which the

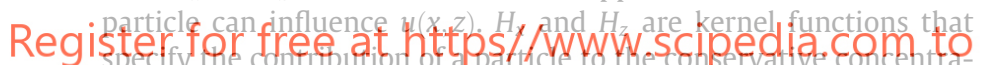
tion $u(x, z, t)$. The shape of the Kernel functions and the size of the support volume $\left\{b_{x}, b_{z}\right\}$ determines the degree of smoothing of concentrations. To be consistent with the layered system (each layer having a small thickness with a spatially uniform hydraulic conductivity), we used a box function for $H_{z}$ with $b_{z}$ equal to the layer thickness and centered at the layer centroid,

$H_{z}\left(d_{z}\right)= \begin{cases}1 / b_{z} & \text { if }\left|d_{z}\right|<1, \\ 0 & \text { Otherwise }\end{cases}$

and a Gaussian kernel function for $H_{x}$,

$H_{x}\left(d_{x}\right)= \begin{cases}\frac{1}{\sqrt{2 \pi} b_{x}} \exp \left(-\frac{d_{x}^{2}}{2}\right) & \text { if }\left|d_{x}\right|<1, \\ 0 & \text { Otherwise }\end{cases}$

Estimation of concentrations by smoothing techniques is troublesome because an optimum choice of the support volume is crucial to overcome the problems of noisy representation of the data and over-smoothing [16]. For this reason, we used an iterative "plugin" algorithm for the selection of $b_{x}$ that minimizes the mean integrated square error of $u$ for each layer. Details of the algorithm are given in Appendix B. In applying this, we note that concentrations $u(x, z, t)$ are estimated over a fine rectangular grid $(\Delta x=0.004 \mathrm{~m}$, $\Delta z=0.04 \mathrm{~m}$ ) to obtain accurate estimates of the forward finite differences of the partial derivatives of $u$. Fig. 7 shows and excellent match between the analytical solutions of $\bar{r}$ and $\sigma_{r}$ and the numerical simulations in the absence of $\alpha_{\mathrm{T}}$. This imbues us with confidence in our computational methodology. In addition, since analytical solutions are given in terms of ensemble moments and
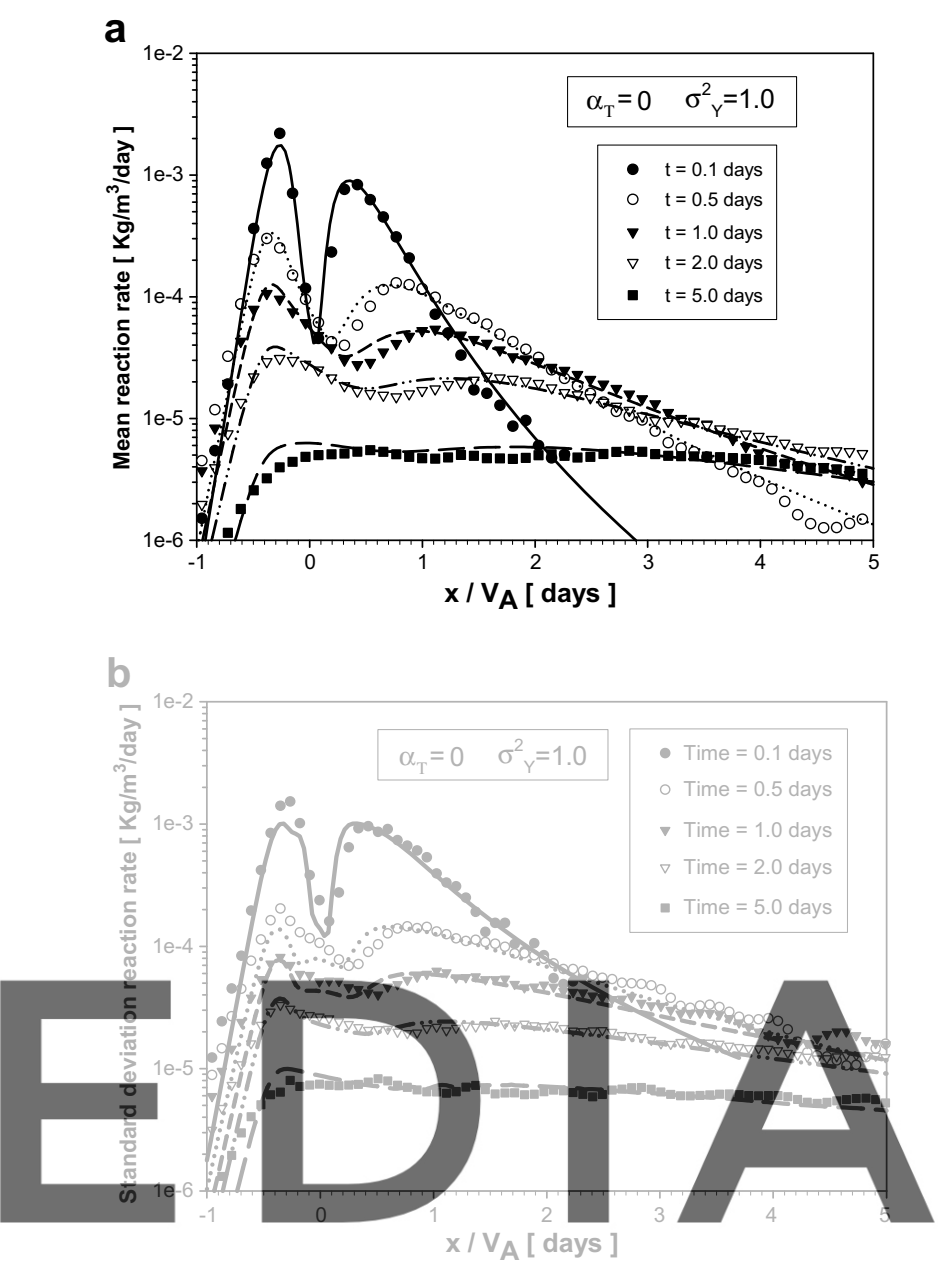

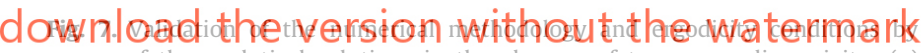
means of the analytical solutions in the absence of transverse dispersivity: (a) ensemble mean reaction rate and (b) reaction rate standard deviation.

numerical simulations represent spatial moments, this corroborates our confidence in the ergodicity assumption adopted.

\subsection{Influence of transverse dispersivity on reaction rates}

Although dispersivity values are typically small at the laboratory scale, local transverse dispersivity has been seen as an important dilution mechanism $[18,20,9]$. Thus, its role in multispecies reactive transport predictions should be carefully assessed. Fig. 8 illustrates the differences in $\hat{r}$ and $\widehat{\sigma}_{r}=\left(\widehat{r}^{2}-\hat{r}^{2}\right)^{1 / 2}$ that are observed in the presence or in the absence of the transverse dispersivity mechanism during transport simulations. The relative importance of the effects of transverse dispersion on the moments of local reaction rates increases with time due to particles undergo substantial mixing along the vertical direction and solute mass exchange between low and high conductivity layers effectively occurs. In order to further discuss the importance of transverse mixing we introduce the following characteristic time, defined as the time needed for a particle to potentially spread vertically over one conductivity integral scale

$\tau_{D}=\frac{\lambda_{z}^{2}}{\bar{D}_{\mathrm{T}}}=\frac{\lambda_{z}^{2}}{\alpha_{\mathrm{T}} K_{\mathrm{A}} J / \phi}$.

Based on this, we employ the term large/small times $\left(t / \tau_{D}\right.$ values $)$ to characterize the amount of local vertical mixing taking place at 

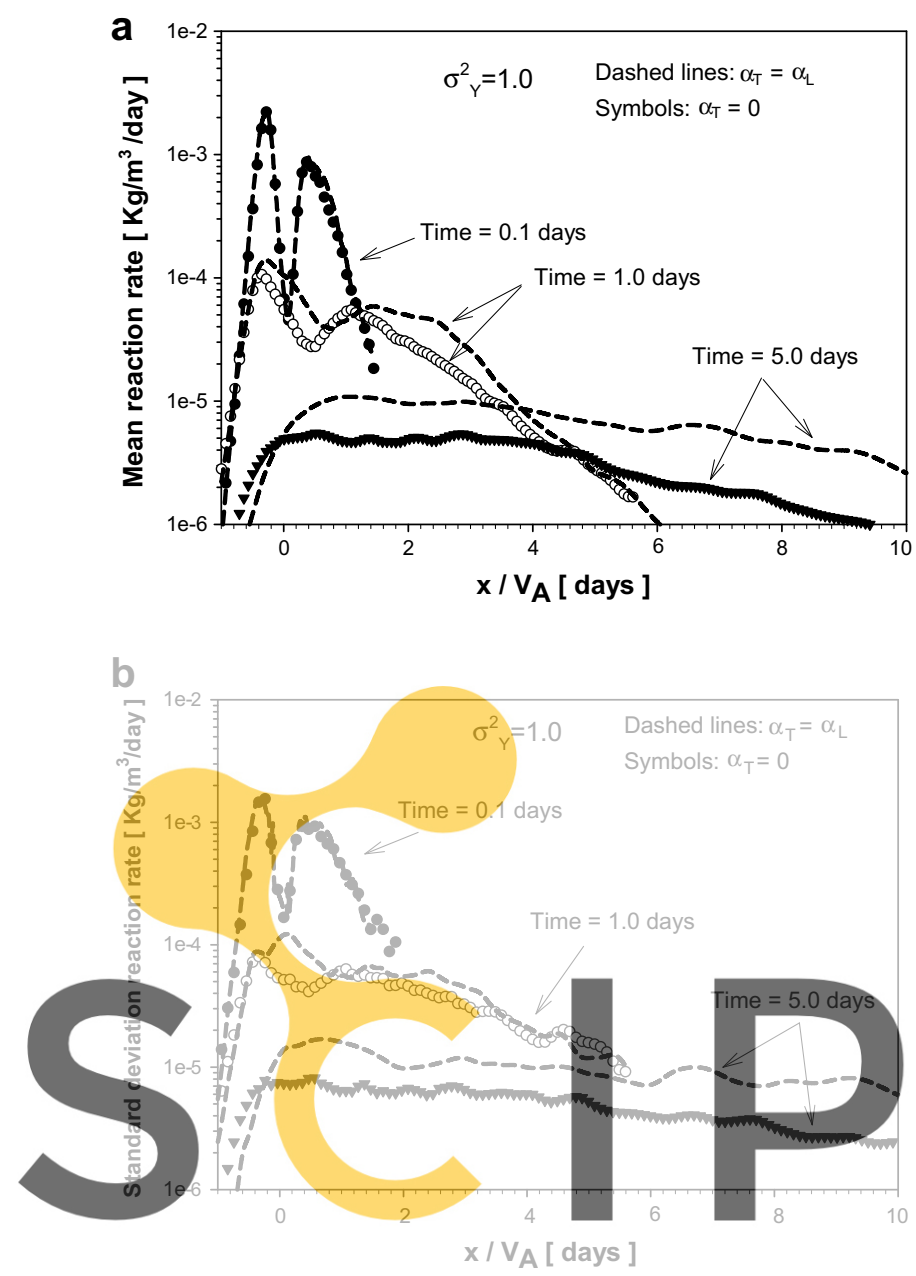

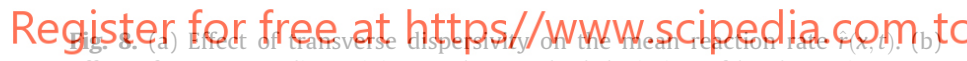
Effect of transverse dispersivity on the standard deviation of local reaction rates $\widehat{\sigma}_{r}(x, t)=\widehat{r}^{2}-\hat{r}^{2}$.

relatively short distances from the source $\left(V_{\mathrm{A}} t<\lambda_{x}\right)$. Noting that $t=5.0$ days corresponds to $t / \tau_{D}=2.1$ in Fig. 8 , it is clear that when transverse mixing is effectively active, the local mean reaction rates are significantly enhanced throughout the aquifer. On the contrary, when $t / \tau_{D}$ is small $\left(t / \tau_{D}=0.04\right.$ for $t=0.1$ days in Fig. 8) particles are still in their initial layer position and there is no actual transverse mixing. At intermediate times $\left(\sim t=1.0\right.$ day or $t / \tau_{D}=0.41$ in Fig. 8), we note that the $\hat{r}$ profiles obtained with and without transverse mixing inherit similar features, i.e., the presence of a double peak, an asymmetric shape, and a pronounced tailing. Nonetheless, transverse mixing still has important consequences, in that it causes not only the mean reaction rate value to increase but also the characteristic time at which reactions take place in the system. This behavior suggests that particles have been transferred from low to high conductive layers where large concentration contrasts in the vertical direction (large transverse gradients) exist, thereby enhancing reaction rates at large distances from the source. Fig. $8 \mathrm{~b}$ displays the effect of transverse mixing on the standard deviation of local reaction rates $\widehat{\sigma}_{r}$. The general behavior is qualitatively similar to what observed for $\hat{r}$, although here the relative effect of transverse mixing is somehow weaker than what observed in Fig. 8a.

Fig. 9 illustrates the relative contribution of the longitudinal and transverse dispersive terms, $r_{\mathrm{L}}$ and $r_{\mathrm{T}}$ in (29), to the mean reaction rate $\hat{r}$ (Fig. 9). As expected, the relative importance of $r_{\mathrm{T}}$ with respect to $r_{\mathrm{L}}$ increases at large distances, where particles have undergone effective vertical mixing and $r_{\mathrm{T}}>r_{\mathrm{L}}$. It is noted that even though transverse mixing reduces concentration gradients, $\nabla u$, suggesting a decrease in reaction rates, the additional contribution given by transverse dispersion counterbalances this effect, ultimately increasing the overall amount of reaction rates in the aquifer as previously shown in Fig. 8.

We now investigate the impact of transverse mixing on the total amount of precipitate in the entire system as a function of time. This is done by analyzing the total mean reaction rate $\widehat{R}(t)$. Fig. 10 compares simulations results for $\widehat{R}(t)$ obtained with and without activating the mechanism of transverse dispersion in the transport simulation processes. Results obtained by the numerical integration of (19), in terms of ensemble average $\bar{R}(t)$, are also reported as a reference. Surprisingly, we note that while the overall effect of transverse mixing is significant for point values of $\hat{r}$, the total amount of precipitate in the entire aquifer is not as sensitive, displaying only slightly larger $\widehat{R}(t)$ values for $\alpha_{T} \neq 0$ and large times than in the absence of $\alpha_{\mathrm{T}}$. The observed fact that the importance of transverse mixing on the (average) total amount of precipitate, $\widehat{R}(t)$, is not as crucial as it is for quantifying $\hat{r}(x, t)$ is consistent with the observation that the characteristic time associated with vertical mixing in aquifers is typically large, while most of the precipitation takes place at early times where higher gradients exist. Moreover, we point out that the late time behavior of $\widehat{R}(t)$ for $\alpha_{\mathrm{T}} \neq 0$ displays a decaying rate relatively smaller than $t^{-3 / 2}$, which was previously derived for $\alpha_{T}=0$. As a side remark, we note that

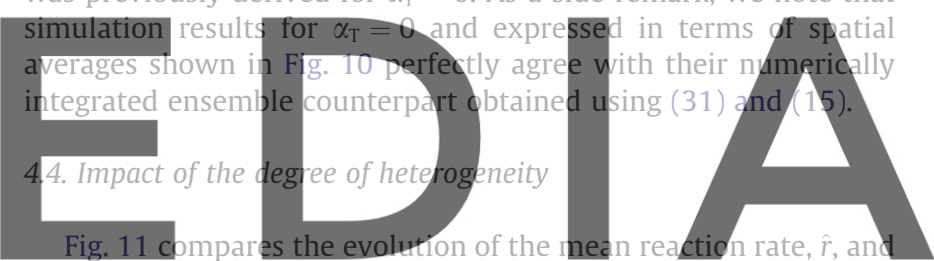

its coefficient of variation, $C V=\widehat{\sigma}_{r} / \hat{r}$, for two different degrees of heterogeneity, $\sigma_{Y}^{2}=1.0$ and $\sigma_{Y}^{2}=2.0$. The latter was obtained by

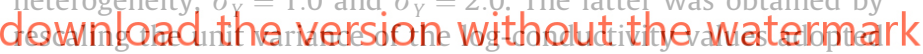
in the previous simulations so that both $K$ fields essentially depict the same specific heterogeneous patterns. Remarkably, Fig. 11 shows that increasing heterogeneity causes two major effects: (a) an overall increase of reaction rates (larger amount of the total reaction rate in the system); and (b) a faster reduction of the double peak observed in the reaction rate distribution. Because $K$ is log-normally distributed, an increase in $\sigma_{Y}^{2}$ yields larger probability to low and high $K$ values. Thus, close to the source, the layered

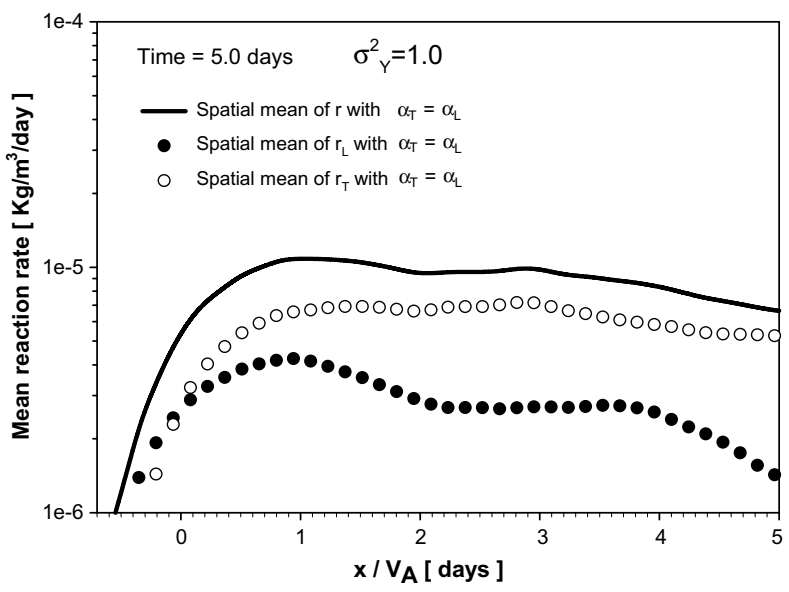

Fig. 9. Separation of the longitudinal and transverse dispersive contributions in the spatial mean reaction rate $\hat{r}(x, t)$, denoted as $r_{\mathrm{L}}$ and $r_{\mathrm{T}}$ in (29). 


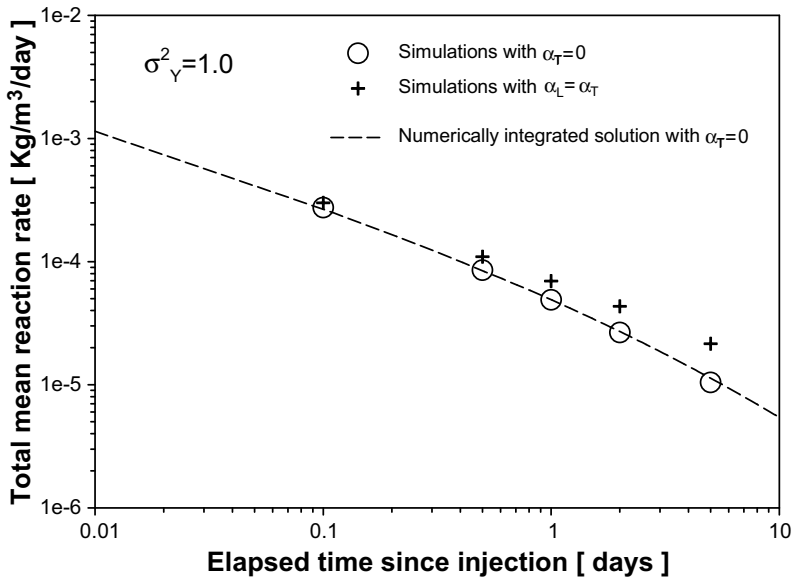

Fig. 10. Effect of transverse dispersivity on the total mean reaction rate. The dashed line displays the ensemble mean reaction rate, $\bar{R}(t)$, obtained by numerical integration of (19), whereas simulation results (symbols) correspond to its spatial average counterpart, $\widehat{R}(t)$.

system incorporates a higher proportion of low conductive layers having large reaction rates, while at the same time produces strong preferential channels carrying chemical reactions further away from the injection. Interestingly, upon comparing Fig. 11a and b

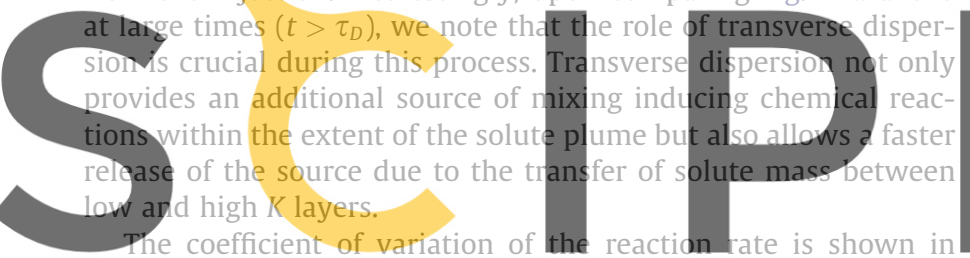
Fig. 11c. Interestingly, we note that uncertainty of large reaction rates decreases with both transverse dispersivity and $\sigma_{Y}^{2}$. This is

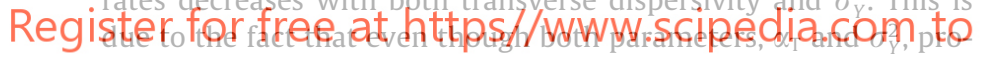
duce an increase of the mean and standard deviation of the reaction rate, the relative amount of increase is less pronounced for the standard deviation.

\subsection{Discussion on effective transport equations}

An interesting feature which can be observed in Figs. 7a and 8a is that at large times $\left(t / \tau_{D} \gg 1\right)$ and in all cases the mean reaction rate profile exhibits a less pronounced double peak with a very long tail. This allows drawing some conclusions about the possibility to describe the mean conservative concentration component, $\bar{u}$, and the associated (ensemble mean) concentrations of reactive species, $\bar{c}_{i}\{i=1,2\}$, by means of an "effective" transport equation. To further analyze this point, we recall first that, for unbounded and non-periodic stratified systems, the effective longitudinal dispersion of a non-reactive solute plume increases with time at large distances displaying a super-diffusive behavior. In the presence of transverse dispersion, this is formally written as $D_{\mathrm{L}}^{\text {eff }}(t) \propto \sqrt{t}$. Based on this, one is tempted to substitute the heterogeneous system by an equivalent homogeneous one by simply incorporating a timevarying dispersion tensor into the ADE. This transport equation can be formally expressed as

$\phi \frac{\partial \tilde{c}_{i}}{\partial t}=-K_{\mathrm{A}} J \frac{\partial \tilde{c}_{i}}{\partial x}+\nabla \cdot\left(\phi \mathbf{D}(t) \nabla \tilde{c}_{i}\right)-\phi \tilde{r}, \quad i=1,2$,

where the wide tilde refers to the equivalent homogeneous media. The application of this model has been studied by several authors for a different type of heterogeneous formation (e.g., [7,33]). Considering (38) and by analogy with (8) the reaction rate would be
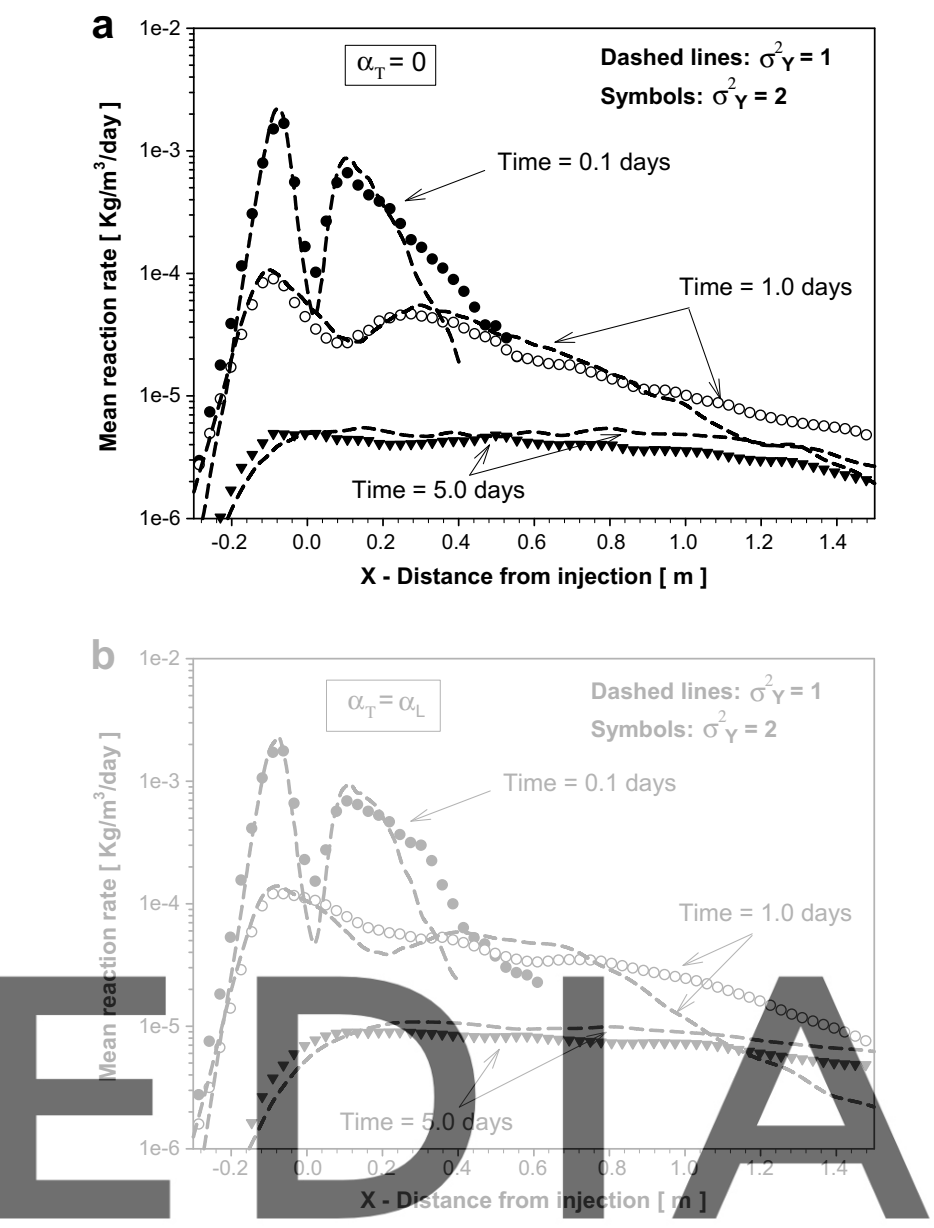

download the dressiopm without the watermark

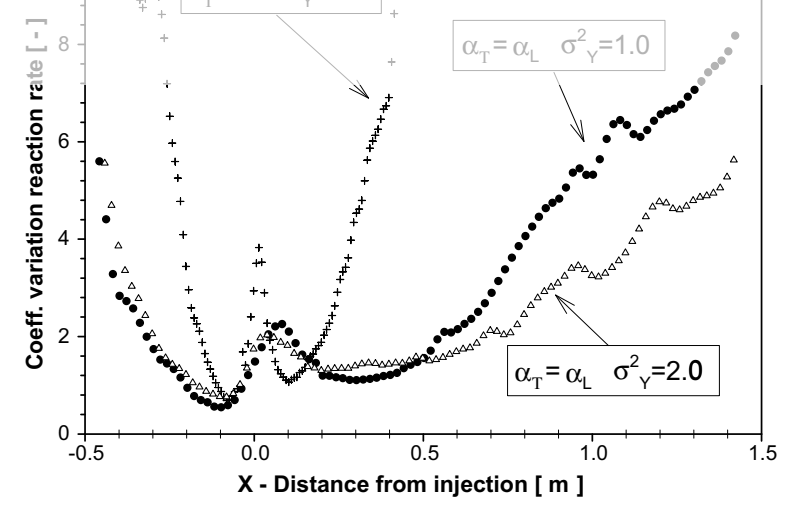

Fig. 11. Impact of the degree of heterogeneity, $\sigma_{Y}^{2}$, on: (a) the mean reaction rate $\hat{r}(x, t)$ for $\alpha_{\mathrm{L}}=\alpha_{\mathrm{T}}$; (b) the mean reaction rate $\hat{r}(x, t)$ for $\alpha_{\mathrm{T}}=0$; and (c) the coefficient of variation of the reaction rate $\mathrm{CV}=\hat{\sigma}(x, t) / \hat{r}(x, t)$ for $\alpha_{\mathrm{L}}=\alpha_{\mathrm{T}}$ and $\alpha_{\mathrm{T}}=0$.

$\tilde{r}=\frac{\partial^{2} \tilde{c}_{2}}{\partial \tilde{u}^{2}} \nabla^{t} \tilde{u} \mathbf{D}(t) \nabla \tilde{u}$

where $\tilde{u}=\tilde{c}_{1}-\tilde{c}_{2}$ is the conservative component governed by

$\phi \frac{\partial \tilde{u}}{\partial t}=-K_{\mathrm{A}} J \frac{\partial \tilde{u}_{i}}{\partial x}+\nabla \cdot\left(\phi \mathbf{D}(t) \nabla \tilde{u}_{i}\right)$.

Solutions to the transport Eq. (40) are capable of preserving the temporal evolution of the second spatial moment of the solute plume as opposed to the use of the classical ADE (constant-in-time dispersivity). Unfortunately, Eq. (39) leads to profiles of mean reac- 
tion rates which are dramatically different from our previous observed solutions in stratified media. To illustrate this, Fig. 12 compares the mean reaction rate distribution of the stratified medium, obtained numerically for $\sigma_{Y}^{2}=1.0$, with its corresponding distribution of $\hat{r}$ generated by using the equivalent transport model (40). Details of the solution of the equivalent model are provided in Appendix C. In agreement with the findings of Luo et al. [24] for isotropic heterogeneous media, the poor reproduction of the reaction rate profile by (40) and (39) directly stems from the inequality $\bar{\nabla}^{t} u \mathbf{D} \nabla u \neq \nabla^{t} \bar{u} \mathbf{D}(t) \nabla \bar{u}$. Here, we further emphasize that the timedependent macrodispersive equation not only generates unrealistic average reaction rates profiles, having two symmetric double peaks (local maxima) and zero values at locations where $\tilde{u}$ displays peak values (and, hence, zero gradients), but also significantly underestimates the total amount of reaction rate (area under the reaction rate distribution) at large times, i.e., for $t>\tau_{D}$. Two important processes responsible for the underestimation of the total reaction rate should be noted: (i) the effective conductivity, defined as the ratio of the mean total flux to the mean head gradient (in this case $K_{\text {eff }}^{\mathrm{F}}=K_{\mathrm{A}}$ ), and the mean reaction rate require a fundamentally different averaging procedure of point conductivities because the influence of low $K$ values is more pronounced for the mean reaction rate (see Section 3.3); and (ii) at large times $\left(t>\tau_{D}\right)$, transverse dispersion generates an important additional source of mixing, enhancing the overall amount of chemical reactions taking place within the entire extent of the solute plume. This process is not well characterized by the given macroscopic transport model because transverse concentration gradients of the conservative component taking place within the extent of the solute plume (large transverse source size) are mostly obscured due to homogenization. The contribution of transverse dispersivity can be seen in Fig. 12 by comparing transport simulations with and without transverse dispersivity. In overall, our results point out the fact that upscaling of reactive transport is formally different from upscaling of conservative transport, which tends to underestimate the total reaction rate taking place in the system.

\section{Summary and conclusions}

We have investigated multispecies reactive transport in randomly heterogeneous stratified aquifers with the aim of analyzing the role of heterogeneity on the spatio-temporal distribution of reaction rates. Analytical solutions of the statistical moments of reaction rates are provided in the presence of negligible transverse

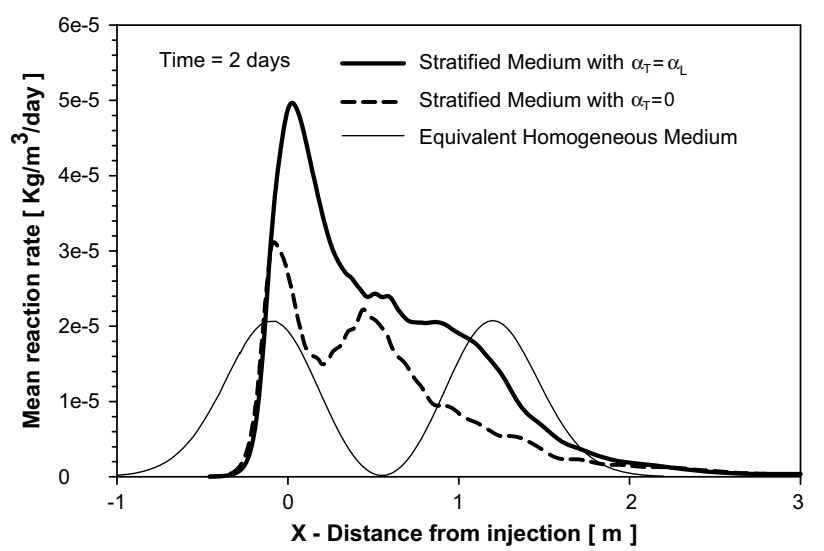

Fig. 12. Comparison between the mean reaction rates observed on the basis of numerical simulations performed within a randomly heterogeneous domain with $\sigma_{Y}^{2}=1.0$ and those calculated according to the equivalent homogeneous solution (39). dispersion. These are seen to be valid for large times and small departures of the injected water from the initial chemical composition, i.e., for large $t / \Delta u_{0}^{2}$ values. Companion numerical simulations were used to explore the effect of transverse mixing and the impact of heterogeneity on the reaction rates. Our work leads to the following key conclusions:

(1) Whereas reaction rates associated with an ADE-based depiction of transport in homogeneous media exhibit a double peak, symmetric profile, and zero reaction rate at a travel distance associated with purely advective processes, reaction rate profiles in heterogeneous media are highly asymmetric having always larger values for the most part. The largest rates are always located in the proximity of the source and their spatial distribution is characterized by long tails. This departure from the behavior typical of homogeneous media increases with heterogeneity, which enhances reaction rates close to the source and far from the average travel distance of the conservative component.

(2) Reaction rates essentially take place in low conductive areas, where large concentration gradients exist. This is in contrast with the nature of effective hydraulic conductivity (associated with the flow problem), which is highly controlled by preferential flow channels, i.e., areas with large $K$ values that concentrate flow. The implication of this result should be emphasized; since usually employed upscaled or macroscopic reactive transport models based on the ADE rely on flow effective conductivities, e.g. those hydraulic conductivities interpreted from pumping tests, the aforementioned discrepancy will cause a systematic underestimation of the total amount of precipitate in the aquifer. In this context, for a randomly heterogeneous stratified system and large times/small departures from the initial composition, we have analytically determined that the effective hydraulic conductivity value required to produce the same total amount of precipitate in an equivalent homogeneous system is consistently smaller than that exclusively associated with the flow problem, $K_{\mathrm{eff}}^{\mathrm{F}}=K_{\mathrm{A}}$, and it is expressed as $K_{\mathrm{eff}}^{\mathrm{R}}=$ $\sqrt{K_{\mathrm{H}} K_{\mathrm{G}}}$

(3) Transverse dispersion causes no variation in the reaction rates at short travel times, and it becomes the dominant process driving reactions at large times and distances from the source. Nonetheless, its effects on the total mean reaction rates are less significant than those observed for the local reaction rates because most of the reaction takes place at small times and close to the source where gradients are higher.

\section{Acknowledgements}

The authors gratefully acknowledge funding by the European Community and ENRESA (Empresa Nacional de Residuos Radioactivos S.A.) through projects GABARDINE and FUNMIG.

\section{Appendix A. Limitations of the approximation}

Most of the previous results for $R(K, t)$ and $\bar{R}(t)$ are based on the simplifying assumption that $u$ can be replaced by $u_{0}$ in the speciation term A in (8) and (9). According to (12) or (28) tends to 0 very rapidly in time. Still, it is important to analyze the range of applicability of the approximation.

Defining the function $f(v)=\left[\left(u_{0}+v\right)^{2}+4 K_{\mathrm{eq}}\right]^{-3 / 2}$, being $v$ the second term in the right-hand-side of (12), the approximation is formally written as $v \rightarrow 0$. Truncating the Taylor's series expansion of $f(v)$ at second order in $v$, 
$f(v)=A^{-3 / 2}\left\{1-\frac{3 u_{0}}{A} v+\left(\frac{15}{2} \frac{u_{0}^{2}}{A^{2}}-\frac{3}{2 A}\right) v^{2}+\ldots\right\}$,

where $A=u_{0}^{2}+4 K_{\text {eq }}$, it follows that we can approximate $f(v)=A^{-3 / 2}$ whenever

$\left|\left(\frac{15 u_{0}^{2}}{2 A^{2}}-\frac{3}{2 A}\right) v^{2}-\frac{3 u_{0}}{A} v\right|<\varepsilon$,

where $\varepsilon$ is a specified tolerance (relative error). By the definition of $f(v)$, the Taylor expansion variable $v$ is a function of the distance from the source, time, and layer properties $K(z)$. Substituting $v$ by $v_{\max }=\Delta u_{0}\left(4 \pi t D_{\mathrm{G}}\right)^{-1 / 2}$ in (42) we obtained a simple conservative criterion for $\bar{R}(t)$, where $D_{\mathrm{G}}=\alpha_{\mathrm{L}} K_{\mathrm{G}} J / \phi$. Here, by conservative meaning that the criterion always yields a relative error of the total mean reaction rate $\bar{R}(t)$ slightly smaller than the tolerance $\varepsilon$. This was verified by numerically integrating the exact solution given by (19). In general, the solution is

$\frac{t}{\Delta u_{0}^{2}}>\frac{1}{4 \pi D_{\mathrm{G}}} \max \left\{\frac{1}{v_{1}^{2}}, \frac{1}{v_{2}^{2}}\right\}$

with

$v_{1}=\frac{u_{0}^{2}+4 K_{\mathrm{eq}}}{12\left(u_{0}^{2}-K_{\mathrm{eq}}\right)}\left(3 u_{0} \pm \sqrt{9 u_{0}^{2}+24 u_{0}^{2} \varepsilon-24 K_{\mathrm{eq}} \varepsilon}\right)$,

$v_{2}=\frac{u_{0}^{2}+4 K_{\mathrm{eq}}}{12\left(u_{0}^{2}-K_{\mathrm{eq}}\right)}\left(3 u_{0} \pm \sqrt{9 u_{0}^{2}-24 u_{0}^{2} \varepsilon+24 K_{\mathrm{eq}} \varepsilon}\right)$.

A closed-form solution can be obtained whenever $u_{0}=0$, corresponding to the case where equilibrium is reached by $c_{1,0}=c_{2,0}$. Then

$\frac{t}{\Delta u_{0}^{2}}>\frac{3}{32 \pi D_{\mathrm{G}} \varepsilon K_{\mathrm{eq}}}$.

A second limiting case would be that of $\left|u_{0}\right| \gg K_{\text {eq }}$, meaning that in the initial (equilibrium) situation one of the species is dominant. In such a case, and assuming the tolerance is small, the time constraint is written as

$\frac{t}{\Delta u_{0}^{2}}>\frac{1}{4 \pi D_{\mathrm{G}}}\left(\frac{u_{0}}{2}+\frac{u_{0}}{3} \varepsilon\right)^{-2}$.

\section{Appendix B. Algorithm to estimate $\boldsymbol{u}$ from the spatial distribution of particles}

The selection of the bandwidth in (36) is the main problem for the kernel estimation of the conservative component concentrations. A compromise is needed to address this problem; oversmoothing produces a bias in the estimation of $u$ whereas undersmoothing generates a noisy representation of the concentrations. Here, to be consistent with the layered system, we used a box function for $H_{z}$ in (33) with $b_{z}$ equal to the layer thickness and centered at the layer centroid. Thus, the concentration of $u$ for a given layer $k$ can be written as

$u_{k}(x, t) \approx u_{k}^{*}(x, t)=\frac{1}{\phi} \sum_{p \in \Omega_{k}} m_{p} H_{x}\left(\frac{x-X_{p}}{b_{x}}\right) \quad \forall k$,

where $\Omega_{k}$ denotes the layer domain. Then, for each layer, the optimal $b_{x}$ in $H_{x}$ was obtained by minimizing the mean integrated square error (MISE) of the distribution of $u_{k}(x, t)$, defined as

$\operatorname{MISE}\left(b_{x} ; t\right)=\left(\frac{\phi}{M}\right)^{2} E\left\{\int\left(u_{k}(x, t)-u_{k}^{*}(x, t)\right)^{2} \mathrm{~d} x\right\}$

where $M=\sum_{p} m_{p}$. Knowing that the same mass is associated to all particles, $M=N_{p} m_{p}$, and defining $K_{x}=b_{x} H_{x}$ and $p_{k}=\phi u_{k} / M$, MISE allows the following asymptotic representation when $b_{x} \rightarrow 0$ and $N_{p} b_{x} \rightarrow \infty$ [16]

$\operatorname{MISE}\left(b_{x} ; t\right) \approx \frac{1}{N_{p} b_{x}}\left\|K_{x}\right\|_{2}^{2}+\frac{1}{4} b_{x}^{4}\left(\mu_{2}\left(K_{x}\right)\right)^{2}\left\|p_{k}^{\prime \prime}\right\|_{2}^{2}$

with

$\|f(x)\|_{2}^{2}=\int f(x)^{2} \mathrm{~d} x$

$\mu_{2}\left(K_{x}\right)=\int x^{2} K_{x}(x) \mathrm{d} x$

$p_{k}^{\prime \prime}=\frac{\phi}{M} \frac{\partial^{2} u_{k}}{\partial x^{2}}$

Setting $\partial($ MISE $) / \partial b_{x}=0$ yields

$b_{x}^{\text {opt }}=\left(\frac{\left\|K_{x}\right\|_{2}^{2}}{\left\|p_{k}^{\prime \prime}\right\|_{2}^{2}\left(\mu_{2}\left(K_{x}\right)\right)^{2} N_{p}}\right)^{1 / 5}$.

Hence, the optimal bandwidth involves the unknown function $p_{k}$ which needs to be further estimated. Here, we used the plug-in method presented by Engel et al. [12], who employed another kernel estimate of $u_{k}^{\prime \prime}$ based on a bandwidth $\beta$ to estimate $\left\|p_{k}^{\prime \prime}\right\|_{2}^{2}$ so that

$\left\|p_{k}\right\|_{2}^{2} \approx R(\beta)=\frac{1}{N_{p}^{2} \beta^{5}} \sum_{i, j} L_{2}\left(\frac{X_{p, i}-X_{p, j}}{\beta}\right)$.

The functional $L_{2}$ is the convolution of $K^{\prime \prime}$ by itself, $L_{2}=K^{\prime \prime} \circ K^{\prime \prime}$. The bandwidth $\beta$ is chosen to be a linear function of $b_{x}$ with the form $\beta\left(b_{x}\right)=C\left(N_{p}\right)^{q} b_{x}, C$ and $q$ are constants. Briefly, the algorithm presented by Engel et al. [12] is as follows: (1) Select an initial bandwidth, $\quad b_{x}^{(0)}=B\left(N_{p}\right)^{-p} ; \quad$ (2) Iterate $i=1, \ldots, I \quad$ with $\beta^{(i-1)}=C\left(N_{p}\right)^{q} b_{x}^{(i-1)}$ and $b_{x}^{(i-1)}=\left(\left\|K_{x}\right\|_{2}^{2} / R\left(\beta^{(i-1)}\right) \mu_{2}^{2}\left(K_{x}\right) N_{p}\right)^{1 / 5}$; and finally, (3) set $b_{x}^{\text {opt }}=b_{x}^{(I)}$. Tuning parameters $\{C, q, p, B, I\}$ required for the implementation of this algorithm are given in [12].

\section{Appendix C. Solution of the equivalent transport model}

The set-up of the equivalent transport problem is the same as that previously described during the design of transport simulations in Section 4.1 with the exception that the heterogeneous $K$ field is replaced by an equivalent homogeneous conductivity, $K_{\text {eff }}^{\mathrm{F}}=K_{\mathrm{A}}$. The effects of heterogeneity are included into the transport model by considering a time-dependent macrodispersion tensor, $\mathbf{D}(t)$, into the advection-dispersion equation (38). Initially, the solute plume extends 100 integral scales in the $z$-direction and groundwater is driven by uniform gradient, $J$, oriented in the $x$ direction. The plume is far enough from the boundaries so that boundary effects can be disregarded. Based on this and using the principal of superposition, the analytical solution of (38) in a two-dimensional system is written as [38]

$$
\begin{aligned}
u(x, z, t)= & \int_{z_{i}}^{z_{f}} u_{0}+\frac{\Delta u_{0}}{2 \pi \sqrt{S_{x x}(t) S_{z z}(t)}} \\
& \times \exp \left(-\frac{\left(x-V_{\mathrm{A}} t\right)^{2}}{2 S_{x x}(t)}-\frac{(z-\zeta)^{2}}{2 S_{z z}(t)}\right) \mathrm{d} \zeta
\end{aligned}
$$

$S_{i j}(t)=2 \int_{0}^{t} D_{i j}(\tau) \mathrm{d} \tau$

where $z_{i}$ and $z_{f}$ defines the initial vertical extent of the solute plume, and we have considered that the principal components of $\mathbf{D}(t)$ are aligned with the $x$ - and $z$-axis. From the analytical solution of $u$, we can directly estimate the reaction rates in the system as

$r(x, z, t)=\frac{\partial^{2} c_{2}}{\partial u^{2}}\left(D_{x x}(t)\left(\frac{\partial u}{\partial x}\right)^{2}+D_{z z}(t)\left(\frac{\partial u}{\partial z}\right)^{2}\right)$, 
where

$$
\begin{aligned}
\frac{\partial u}{\partial x}= & -\frac{\Delta u_{0}\left(x-V_{\mathrm{A}} t\right)}{2 \pi S_{x x}(t) \sqrt{S_{x x}(t) S_{z z}(t)}} \exp \left(-\frac{\left(x-V_{\mathrm{A}} t\right)^{2}}{2 S_{x x}(t)}\right) \\
& \times \int_{z_{i}}^{z_{f}} \exp \left(-\frac{(z-\zeta)^{2}}{2 S_{z z}(t)}\right) \mathrm{d} \zeta
\end{aligned}
$$

$$
\begin{aligned}
\frac{\partial u}{\partial z}= & -\frac{\Delta u_{0}}{2 \pi \sqrt{S_{x x}(t) S_{z z}(t)}} \exp \left(-\frac{\left(x-V_{\mathrm{A}} t\right)^{2}}{2 S_{x x}(t)}\right) \\
& \times \int_{z_{i}}^{z_{f}} \frac{z-\zeta}{S_{x x}(t)} \exp \left(-\frac{(z-\zeta)^{2}}{2 S_{z z}(t)}\right) \mathrm{d} \zeta .
\end{aligned}
$$

Knowing the reaction rate at any given location, we can calculate the mean reaction rate as

$\hat{r}(x, t)=\frac{1}{z_{f}-z_{i}} \int_{z_{i}}^{z_{f}} r(x, z, t) \mathrm{d} z$.

Hence, the mean reaction rate is fully defined once the $S_{i j}(t)$ coefficients are known. These coefficients were estimated using the relationship of $S_{i j}(t)$ with the spatial moments of the solute plume, i.e., $S_{i j}(t)=\sum_{i j}(t)-\sum_{i j}(t=0)$, where $\sum_{i j}(t)$ is the second spatial moment about the center of mass of the solute plume of $u$, defined as

$\sum_{i j}(t)=\frac{\phi}{M} \int_{\mathfrak{R}^{2}}\left(x_{i}-x_{g, i}(t)\right)\left(x_{j}-x_{g . j}(t)\right) u(\mathbf{x}, t) \mathrm{d} \mathbf{x}$,

$x_{g, i}$ being the coordinates of the plume center of mass,

$x_{g, i}(t)=\frac{\phi}{M} \int_{\mathfrak{R}^{2}} x_{i} u(\mathbf{x}, t) \mathrm{d} \mathbf{x}$.

These were numerically calculated from the spatial distribution of particles obtained previously during the transport simulations in stratified media presented in Section 4.1 as

$\sum_{i j}(t) \approx \frac{1}{N_{p}} \sum_{p \in N_{p}} X_{p, i}(t) X_{p, j}(t)-x_{g, i}(t) x_{g, j}(t)$

$x_{g, i}(t) \approx \frac{1}{N_{p}} \sum_{p \in N_{p}} X_{p, i}(t)$

where $\mathbf{X}_{p}$ is the particle position vector, and $N_{p}$ is the number of particles in the system.

\section{References}

[1] Bear J. Dynamics of fluids in porous media. New York: Elsevier; 1972.

[2] Bellin A, Rinaldo A. Analytical solutions for transport of linearly adsorbing solutes in heterogeneous formations. Water Resour Res 1995;31(6):1505-11.

[3] Carrera J, Vázquez-Suné E, Castillo O, Sanchez-Vila X. A methodology to compute mixing ratios with uncertain end-members. Water Resour Res 2004;40:W12101. doi:10.1029/2003WR002263.

[4] Cvetkovic VD, Shapiro AM. Solute advection in stratified formations. Water Resour Res 1989;25:1283-90.

[5] Cvetkovic V, Dagan G, Cheng H. Contaminant transport in aquifers with spatially variable hydraulic and sorption properties. Proc Roy Soc London Ser A 1998;454:1-36.

[6] Dagan G. Flow and transport in porous formations. Berlin: Springer-Verlag; 1989.

[7] Dagan G. Upscaling of dispersion coefficients in transport through heterogeneous porous formations. In: Computational methods in water resources X. Norwell Mass: Kluwer Academic; 1994. p. 431-9.

[8] Dagan G, Neuman SP, editors. Subsurface flow and transport. New York: Cambridge University Press; 1997.

[9] De Simoni M, Carrera J, Sanchez-Vila X, Guadagnini A. A procedure for the solution of multicomponent reactive transport problems. Water Resour Res 2005;41:W11410. doi:10.1029/2005WR004056.

[10] De Simoni M, Sanchez-Vila X, Carrera J, Saaltink MW. A mixing ratios-based formulation for multicomponent reactive transport. Water Resour Res, in press. doi:10.1029/2006WR005256
[11] Dykaar BB, Kitanidis PK. Macrotransport of a biologically reacting solute through porous media. Water Resour Res 1996;32(2):307-20.

[12] Engel J, Herrmann E, Gasser T. An iterative bandwidth selector for kernel estimation of densities and their derivatives. Nonparametric Stat 1994;4:21-34.

[13] Fernàndez-Garcia D, Illangasekare TH, Rajaram $H$. Differences in the scale dependence of dispersivity estimated from temporal and spatial moments in chemically and physically heterogeneous porous media. Adv Water Resour 2005;28:745-59.

[14] Fernàndez-Garcia D, Illangasekare TH, Rajaram H. Assessment of the predictive capabilities of stochastic theories in a three-dimensional laboratory tes aquifer: effective hydraulic conductivity and temporal moments of breakthrough curves. Water Resour Res 2005;41:W04002. doi:10.1029 2004WR003523.

15] Gelhar LW. Stochastic subsurface hydrology. New Jersey: Prentice Hall; 1993.

[16] Hardle W. Smoothing techniques with implementation in S. New York: Springer-Verlag; 1990

[17] Hu BX, Deng F-W, Cushman JH. Nonlocal reactive transport with physical and chemical heterogeneity: linear nonequilibrium sorption with random $\mathrm{Kd}$ Water Resour Res 1995;31(9):2239-52.

[18] Kapoor V, Gelhar LW. Transport in three-dimensionally heterogeneous aquifers: 1. Dynamics of concentration fluctuations. Water Resour Res 1994;30(6):1775-88.

[19] Kinzelbach W. The random walk method in pollutant transport simulations In: Custodia E, Gurgui A, Lobo Ferreira JP, editors. Groundwater flow and quality modelling. NATO ASI series C Mathematical and Physical Sciences, vol. 224. Norwell, (MA): Reidel; 1988. p. 227.

[20] Kitanidis PK. The concept of the dilution index. Water Resour Res 1994;30(7):2011-26.

[21] Kitanidis PK. Introduction to geostatistics: applications in hydrogeology. New York: Cambridge University Press; 1997.

[22] Lawrence AE, Sanchez-Vila X, Rubin Y. Conditional moments of the breakthrough curves of kinetically sorbing solute in heterogeneous porous media using multirate mass transfer models for sorption and desorption. Water Resour Res 2002;38(11):1248. doi:10.1029/2001WR001006.

[23] Lichtner PC, Tartakovsky DM. Stochastic analysis of effective rate constant for heterogeneous reactions. Stoch Environ Res Risk Assess 2003;17:419-29.

[24] Luo J, Dentz M, Carrera J, Kitanidis P. Effective reaction parameters for mixing controlled reactions in heterogeneous media. Water Resour Res 2008;44:W02416. doi:10.1029/2006WR005658.

[25] Matheron G, De Marsily G. Is transport in porous media always diffusive? A counterexample. Water Resour Res 1980;16(5):901-17.

[26] Meile C, Kagan T. Scale dependence of reaction rates in porous media. Adv Water Resour 2006;29:62-71.

[27] Mercado A. The spreading pattern of injected water in a permeability stratified aquifer. IAHS AISH Publ 1967;72:23-36.

[28] Persefoni EK, Tsimpanogiannisa IN, Yortsos CY, Lichtner PC. On the upscaling of reaction-transport processes in porous media with fast or finite kinetics. Chem Eng Sci 2002;57:2565-77.

[29] Quinodoz HAM, Valocchi AJ. Stochastic analysis of the transport of kinetically sorbing solutes in aquifers with randomly heterogeneous hydraulic conductivity. Water Resour Res 1993;29(9):3227-40.

[30] Rajaram H. Time and scale dependent effective retardation factors in heterogeneous aquifers. Adv Water Resour 1997;20(4):217-30.

[31] Reichle R, Kinzelbach W, Kinzelbach H. Effective parameters in heterogeneous and homogeneous transport models with kinetic sorption. Water Resour Res 1998;34(4):583-94.

[32] Rubin Y. Applied stochastic hydrogeology. Oxford University Press; 2003.

[33] Rubin Y, Sun A, Maxwell R, Bellin A. The concept of block effective macrodispersivity and a unified approach for grid-scale- and plume-scaledependent transport. J Fluid Mech 1999;395:161-80.

[34] Saaltink MW, Batlle F, Ayora C, Carrera J, Olivella S. RETRASO, a code for modeling reactive transport in saturated and unsaturated porous media. Geo Acta 2004:2(3):235-51.

[35] Salamon P, Fernàndez-Garcia D, Gómez-Hernández JJ. A review and numerica assessment of the random walk particle tracking method. J Contam Hydro 2006;87:277-305

[36] Salamon P, Fernàndez-Garcia D, Gómez-Hernández JJ. Modeling trace transport at the MADE site: the importance of heterogeneity. Water Resour Res 2007;43:W08404. doi:10.1029/2006WR005522.

[37] Sanchez-Vila X, Rubin Y. Travel time moments for sorbing solutes in heterogeneous domains under nonuniform flow conditions. Water Resour Res 2003;39(4):1086. doi:10.1029/2002WR001399.

[38] Sposito G, Barry DA. On the Dagan model of solute transport in groundwater: fundamental aspects. Water Resour Res 1987;23(10):1867-75.

[39] Tompson AFB, Gelhar LW. Numerical simulation of solute transport in threedimensional, randomly heterogeneous porous media. Water Resour Res 1990:26(10):2541-62.

[40] Zinn B, Harvey CF. When good statistical models of aquifer heterogeneity go bad: a comparison of flow, dispersion, and mass transfer in connected and multivariate Gaussian hydraulic conductivity fields. Water Resour Res 2003;39(3):1051. doi:10.1029/2001WR001146. 